\title{
Salivary bacterial signatures in depression-obesity comorbidity are associated with neurotransmitters and neuroactive dipeptides
}

Suzi Hong ( $\sim$ s1hong@health.ucsd.edu )

University of California San Diego

Gajender Aleti

University of California San Diego

Jordan N. Kohn

University of California San Diego

Emily A. Troyer

University of California San Diego

Kelly Weldon

University of California San Diego

Shi Huang

University of California San Diego

Anupriya Tripathi

University of California San Diego

Pieter C. Dorrestein

University of California San Diego

Austin D. Swafford

University of California San Diego

Rob Knight

University of California San Diego

Research article

Keywords: depressive symptomatology, obesity, depressive symptomatology-obesity comorbidity, symptomatology independently

Posted Date: August 23rd, 2021

DOl: https://doi.org/10.21203/rs.3.rs-829379/v1

License: (c) (1) This work is licensed under a Creative Commons Attribution 4.0 International License.

Read Full License 



\section{Title page}

2 Salivary bacterial signatures in depression-obesity comorbidity are associated with 3 neurotransmitters and neuroactive dipeptides

4 Gajender Aleti ${ }^{1}$ galeti@health.ucsd.edu, Jordan N. Kohn¹ jokohn@health.ucsd.edu, Emily A.

5 Troyer ${ }^{1}$ etroyer@health.ucsd.edu, Kelly Weldon ${ }^{3,6}$ kcweldon@eng.ucsd.edu, Shi Huang ${ }^{2,3}$ 6 shh047@health.ucsd.edu, Anupriya Tripathi ${ }^{2,6}$ anupriya.tripathi92@gmail.com, Pieter C.

7 Dorrestein ${ }^{2,3,6,7}$ pdorrestein@health.ucsd.edu, Austin D. Swafford ${ }^{3}$ adswafford@eng.ucsd.edu, 8 Rob Knight ${ }^{2,3,4,5}$ robknight@eng.ucsd.edu, Suzi Hong*1,8 s1hong@health.ucsd.edu

\section{Affiliations}

$10{ }^{1}$ Department of Psychiatry, University of California San Diego, La Jolla, CA 92093, United

11 States of America.

$12{ }^{2}$ Department of Pediatrics, University of California San Diego, La Jolla, CA 92093, United 13 States of America.

$14{ }^{3}$ Center for Microbiome Innovation, University of California San Diego, La Jolla, CA 92093, 15 United States of America.

$16{ }^{4}$ Department of Computer Science and Engineering, University of California San Diego, La 17 Jolla, CA 92093, United States of America.

$18{ }^{5}$ Department of Bioengineering, University of California San Diego, La Jolla, CA 92093, United 19 States of America. 
$20{ }^{6}$ Skaggs School of Pharmacy and Pharmaceutical Sciences, University of California San Diego,

21 La Jolla, CA 92093, United States of America.

$22{ }^{7}$ Collaborative Mass Spectrometry Innovation Center, University of California San Diego, La 23 Jolla, CA 92093, United States of America.

$24{ }^{8}$ Herbert Wertheim School of Public Health and Longevity Science, University of California San 25 Diego, La Jolla, CA 92093, United States of America.

$26 *$ Corresponding author: Suzi Hong, PhD; s1hong@health.ucsd.edu

\section{Abstract}

\section{Background}

29 Depression and obesity, both of which are highly prevalent and inflammation underlies, often co30 occur. Microbiome perturbations are implicated in obesity-inflammation-depression

31 interrelationships, but how microbiome alterations contribute to underlying pathologic processes

32 remains unclear. Metabolomic investigations to uncover microbial neuroactive metabolites may 33 offer mechanistic insights into host-microbe interactions.

\section{Methods}


35 Using $16 \mathrm{~S}$ sequencing and untargeted mass spectrometry of saliva, and blood monocyte

36 inflammation regulation assays, we determined key microbes, metabolites and host inflammation

37 in association with depressive symptomatology, obesity, and depressive symptomatology-obesity

38 comorbidity.

\section{Results}

40 Gram-negative bacteria with inflammation potential were enriched relative to Gram-positive

41 bacteria in comorbid obesity-depression, supporting the inflammation-oral microbiome link in

42 obesity-depression interrelationships. Oral microbiome was highly predictive of depressive

43 symptomatology-obesity co-occurrences than obesity and depressive symptomatology

44 independently, suggesting specific microbial signatures associated with obesity-depression co-

45 occurrences. Mass spectrometry analysis revealed significant changes in levels of signaling

46 molecules of microbiota, microbial or dietary derived signaling peptides and aromatic amino

47 acids among host phenotypes. Furthermore, integration of the microbiome and metabolomics

48 data revealed that key oral microbes, many previously shown to have neuroactive potential, co-

49 occurred with potential neuropeptides and biosynthetic precursors of the neurotransmitters

50 dopamine, epinephrine and serotonin.

\section{Conclusions}

52 Together, our findings offer novel insights into oral microbial-brain connection and potential 53 neuroactive metabolites involved. 


\section{Background}

55 Depression and obesity are common, debilitating, and frequently co-occurring chronic conditions

56 with increasing incidences globally [1]. Nearly 39\% of the adult population are overweight and

$5713 \%$ are obese worldwide (WHO, 2016), while $5 \%$ of the world population are affected by mood

58 disorders (WHO, 2017) [2,3]. The relationship between obesity and depression is often

59 bidirectional [4], as prevalence of depression among individuals with obesity is significantly

60 higher than that in the general population $[5,6]$. Conversely, individuals with depression are more

61 likely to develop obesity compared to non-depressed individuals [7]. Despite the advent of

62 antidepressant drugs and their long-term usage in clinical treatment, the majority of patients with

63 depression are treatment-refractory, and obesity may further reduce the efficacy of

64 antidepressants [8]. Furthermore, comorbid depression and obesity are strongly associated with

65 several diseases such as type 2 diabetes mellitus, cardiovascular diseases, chronic kidney disease

66 and cancer, reducing both longevity and quality of life [2,9]. Therefore, obesity and depression,

67 and their co-occurrence, pose a major public health concern worldwide.

68 Inflammatory dysregulation is a common pathogenic mechanism underlying the co-

69 occurrence of depression and obesity, as both are associated with chronic low-grade

70 inflammation [10,11]. Individuals with obesity and depression evidence increased concentrations

71 of peripheral and central inflammatory cytokines and acute phase reactants, such as interleukin

72 (IL)-6, tumor necrosis factor alpha (TNF- $\alpha$ ), and C-reactive protein (CRP) $[11,12]$. In obesity,

73 macrophages accumulate in adipose tissue leading to local and systemic inflammation $[13,14]$,

74 which can contribute to depressive symptoms via multiple mechanisms, such as by decreasing

75 neurotransmitter availability, and by potentiating neuroinflammatory processes such as 
microglial activation and peripheral monocyte trafficking to the central nervous system (CNS) $[10,15,16]$. It should be noted, however, that inflammation has been shown to underlie only a subset of depression cases [17], hence the conceptualization of a theoretical immuno-metabolic subtype of major depressive disorder [18]. Nonetheless, inflammatory dysregulation remains a central mechanism underlying the co-occurrence of depression and obesity, and this is likely relevant to sub-clinical depressive symptomatology. To this end, our previous work has demonstrated that even in individuals without clinical diagnosis of depression, higher depressive symptom scores, obesity, and downregulated glucocorticoid and adrenergic receptor-mediated cellular inflammatory control are interrelated [19-21].

Although psychological stress, host genetics and environmental factors have been shown to contribute to obesity and depression, recently, the human microbiome (i.e., collection of diverse microorganisms and their genetic material) and metabolome (i.e., a large collection of structurally diverse metabolites) have been implicated in processes of energy homeostasis, mood and behavior, and immune regulation, and may therefore offer a novel mechanism underlying the co-occurrence of depression and obesity [2]. Animal studies of obesity have shown that depletion of members of Bifidobacterium, Lactobacillus, and Akkermansia are associated with weight gain, increased inflammation, increased depressive behavior and changes in neural circuitry [22,23]. Animal studies have also shown that increased permeability in the intestinal barrier and the blood-brain barrier (BBB) are associated with increased plasma lipopolysaccharide (LPS) levels [22-24] and neuroinflammation [23]. Altogether, these studies suggest that increased intestinal barrier permeability and subsequent translocation of gut bacterial endotoxin, particularly LPS from Gram-negative bacterial cell walls, into systemic circulation, is a source of chronic low- 
grade inflammation and metabolic endotoxemia, which can potentiate neuroinflammatory processes, and therefore serve as a potential mechanism underlying the occurrence of depressive symptoms in the context of obesity. However, this remains to be established in humans.

It is to be noted that human microbiome studies in depression and obesity, and indeed in health and disease, have focused largely on the ecosystem of the distal gut, while few studies have examined the microbial ecology of the oral cavity outside of oral-related conditions such as dental caries (i.e., tooth decay) and periodontitis (i.e., severe gum inflammation). The oral cavity, an entry portal to both the digestive and respiratory tracts, contains the most diverse microbial community after the gut, harboring more than 700 unique bacterial species with at least 150 specialized bacterial species per mouth $[25,26]$. More than $60 \%$ of the microbial species found in the oral cavity have been shown to be potentially transmitted to the gut, suggesting that oral cavity is a reservoir for gut microbial strains in shaping the gut microbiome in health and disease [27]. Dysregulation of the unique microbe-microbe and microbe-host interactions in the oral ecosystem has been associated with systemic inflammatory diseases such as inflammatory bowel syndrome $[28,29]$ beyond an array of oral diseases. In addition, oral microbiota have also been associated with several neurological diseases, such as Alzheimer's disease (AD) [30], multiple sclerosis [31] and Parkinson's disease [32]. Previously, our group found that salivary microbial diversity and diurnal variability were associated with both peripheral proinflammatory cytokine levels and psychological distress in this cohort on which this study is based [33]. The intimate link between the oral microbiota and systemic human diseases, as evidenced by aforementioned studies suggests that the oral cavity is likely a promising site for gaining insight into the pathophysiology of depression-obesity comorbidity. Moreover, the oral cavity is easily 
accessible via non-invasive as well as 'on-demand' collection of saliva samples for multi-omics

121 applications.

122 While mechanisms linking the oral microbiota to the brain (i.e. "oral-brain axis") remain

123 largely unknown [34,35], recent studies have speculated several transmission routes of how oral

124 bacteria may reach the brain and influence neuro-immune activity and inflammation [36]. For

125 instance, routine dental procedures such as flossing, brushing and cleaning may cause oral

126 bacteria to enter the blood circulation and cause bacteremia [37], and some of these microbes

127 may traverse the BBB. Alteration in the permeability of the BBB may also expose the brain to

128 bacterial metabolites triggering an inflammatory response, which in turn alters functioning of the

129 CNS. For example, Porphyromonas gingivalis, a resident oral bacterium and a keystone

130 pathogen in periodontitis has been found in the brain of AD patients [30] as well as neurotoxic

131 proteases i.e., gingipains produced by $P$. gingivalis [30].

132 A recent study has shown that human gut bacteria encode at least 56 gut-brain metabolic

133 pathways, which encompass both known and novel microbial pathways for synthesis and

134 degradation of a number of neurotransmitters that have potential to cross the intestinal barrier

135 and BBB [35]. A subset of these gut-brain pathway effectors, for instance dopamine, glutamate,

136 tryptophan and gamma-aminobutyric acid (GABA) were either enriched or depleted in patients

137 with major depression [35]. In particular, tryptophan metabolic pathways have been shown to be

138 widely distributed across human gut bacterial species [35]. Intriguingly, the majority of these gut

139 bacterial species with neuroactive potential are also found to be residents of the oral cavity [25].

140 However, to what extent these bacterial species can truly biosynthesize neurotransmitters within

141 the host, either in the gut or the oral cavity, remains unknown. Thus, utilization of metabolomics 
142 offers a functional readout of both host and microbial phenotypes encoded in the genome

143 [38,39], and in conjunction with microbiome analyses, can provide mechanistic insights, yet

144 current knowledge is greatly limited. In particular, microbial specialized metabolites have been

145 shown to be canonical mediators of microbe-microbe and microbe-host interactions, and the

146 most predominant specialized metabolites are of great interest for understanding the mechanisms

147 of these interactions at the molecular level [38-40]. In this regard, the vast and highly diverse

148 array of short peptides shown to play key roles in bacterial cell signaling [41], immune

149 modulation, and neuroactive metabolism [42-44] remains largely unexplored. A recent study has

150 shown that depletion of a variety of structurally uncharacterized dipeptides are associated with

151 inflammatory bowel disease, a chronic inflammatory condition of the gastrointestinal tract [45].

152 These observations prompted us to hypothesize that neurotransmitters and dipeptides likely have

153 pivotal roles in obesity-inflammation-depression interrelationships.

154 In this study we aimed to investigate whether oral microbiota and small-molecule

155 mediators of key microbe-microbe and microbe-host interactions differ by depressive

156 symptomatology and obesity as well as their co-occurrence, and are influenced by inflammatory

157 processes. We performed $16 \mathrm{~S}$ rRNA gene-based sequencing of the oral microbiome and

158 untargeted mass spectrometry of small-molecules from saliva, as well as host inflammation

159 regulation profiles in blood from 60 participants.

\section{Methods}

\section{Participants}


162 A total of 60 lean to obese participants (20-65 years old) with a range of subclinical depressive

163 symptoms, participating in a larger study investigating the impact of obesity on vascular

164 inflammation and immune cell activation in normotension versus stage 1 hypertension (Basal

165 systolic blood pressure (BP): 130-140 mmHg and diastolic BP: 80-90 $\mathrm{mmHg}$ ), were included in

166 this study and provided saliva samples. Participant inclusion/exclusion criteria were previously

167 described in detail [33]. Briefly, participants were excluded if they had diabetes, recent history of

168 smoking or substance abuse, history of cardiovascular disease, history of bronchospastic

169 pulmonary disease, inflammatory disorders or health-related factors affecting immune function,

170 psychosis, major depressive disorder, and stage 2 clinical hypertension or with average BP

$171 \geq 145 / 90 \mathrm{mmHg}$ measured at the lab visit from six measurements on two separate days, using a

172 Dinamap Compact BP monitor (Critikon, Tampa, FL). Sociodemographic characteristics (i.e.,

173 age, sex, and race) and anthropometrics (i.e., height, weight, hip and waist circumference) data

174 were collected.

\section{Obesity characterization}

176 BMI was calculated based on height and weight measurements $\left(\mathrm{kg} / \mathrm{m}^{2}\right)$, and individuals were

177 dichotomized into two groups, based on our prior findings of little notable differences in

178 inflammatory or depressive symptoms state between lean and overweight individuals (ref): non-

179 obese $\left(\mathrm{BMI}<30 \mathrm{~kg} / \mathrm{m}^{2}\right)$ and obese $\left(\mathrm{BMI} \geq 30 \mathrm{~kg} / \mathrm{m}^{2}\right)$. For further adiposity characterization dual

180 x-ray absorptiometry was performed to calculate \%total and trunk body fat.

\section{Depressive symptomatology assessment}


182 Depressive symptoms were assessed using the Beck Depression Inventory (BDI-Ia), a 183 comprehensive and clinically robust self-report 21-item questionnaire (Beck et al., 1996). Each

184 question was scored from 0-3, summed to a BDI total score (BDI-T), and then subcategorized

185 into cognitive-affective (BDI-C) and somatic (BDI-S) depression scores based on the items such

186 as BDI-C: guilt, pessimism and BDI-S: fatigue, sleep disruption [46].

187 Based on obesity status and mean BDI-T scores, participants were categorized into 4188 groups: non-obese and lower-depressive controls ( $N=10$ participants; $n=43$ samples; "controls"), 189 obese and lower-depressive ( $\mathrm{N}=18 ; \mathrm{n}=74 ;$ "Ob/lower-Dep"), non-obese and higher-depressive 190 symptoms ( $\mathrm{N}=5 ; \mathrm{n}=22$; "Non-ob/higher-Dep"), and obese and higher-depressive symptoms 191 (N=27; n=122; “Ob/higher-Dep”).

\section{Blood collection and cellular inflammation assay}

193 For detailed protocol, see Supplementary Materials and Methods section. Briefly, LPS194 stimulated blood was incubated with beta-adrenergic receptor agonist isoproterenol and 195 evaluated for intracellular monocyte TNF- $\alpha$ production using flow cytometry, as previously 196 described [47]. Monocyte beta-adrenergic receptor-mediated inflammation control (i.e., 197 "BARIC", a measure of systemic inflammation) was calculated as the arithmetic difference in 198 \%TNF- $\alpha$-producing monocytes between LPS + media-treated and LPS + isoproterenol-treated 199 samples.

\section{Saliva collection, DNA extraction and 16S sequencing}


201 For detailed protocols of saliva collection procedure and 16S analysis, see Supplementary 202 Materials and Methods section. Saliva from each participant was collected at five time points 203 across a single day: waking, mid-morning (10:00 hrs), midday (12:00 hrs), afternoon (14:00 hrs), 204 and evening (17:00 hr).

\section{Statistical analyses}

206 Statistical analyses were conducted using R software (version 3.6.3) in RStudio (version 207 1.2.5019). First, associations among continuous and categorical metadata variables i.e., age, 208 obesity (BMI, \%total body fat and trunk fat), BARIC, BDI scores (BDI-T, BDI-C and BDI-S)

209 were assessed using univariate Spearman correlations across all participants using psych package 210 in R software. We applied a simple linear mixed-effects model (LMM) fit to model two alpha 211 diversity measures (Shannon index and Faith's PD) using restricted maximum likelihood

212 (REML) with a random intercept by participant to account for repeated measurements across the 213 day, and main effects of obesity status, depressive symptom status, and BARIC. Age, sex, race 214 were included as covariates in the model. Beta-diversity between groups was tested using non215 parametric PERMANOVA with 999 permutations constrained by participant to adjust for 3-5 216 samples per participant, and a test of homogeneity of dispersion was conducted with the same 217 constraints using PERMDISP2 in vegan package to test overall species composition differences 218 within the groups. Next, post-hoc pairwise comparison was performed using pairwiseAdonis.

\section{Random forest classifications}


220 A random forest sample classifier was trained based on the 16S data with tuned hyperparameters

221 (num.trees $=500$, mtry $=45$ ) in the 20-time repeated, stratified 5-fold cross-validation using caret 222 package in $\mathrm{R}$ software. The dataset was repeatedly split into five groups with similar class 223 distributions, and we trained the classifier on $80 \%$ of the data, and made predictions on the 224 remaining $20 \%$ of the data in each fold iteration. We next evaluated the performance of the 225 classifier on predicting the four groups (i.e. controls, Ob/lower-Dep, Non-ob/higher-Dep, $226 \mathrm{Ob} /$ higher-Dep) using both area under the receiver operating characteristic curve (AUROC) and 227 area under the precision-recall curve (AUPRC) based on the samples' predictions in the holdout 228 test set using PRROC package in R. To account for multiple samples per-participant, we next performed 20-time repeated group 3-fold cross-validation, where each participant is in a different testing fold and also samples from the same subjects are never in both testing and training folds.

\section{Small molecule metabolite detection through mass spectrometry}

232 Saliva was dried and resuspended in $80 \% \mathrm{MeOH}-20 \%$ water and submitted to untargeted 233 LC/MS/MS analysis. For a detailed protocol, see Supplementary Materials and Methods section.

234 To examine the metabolic potential in the oral ecosystem and understand the intimate link 235 between salivary microbiota and metabolome in obesity-depressive symptom relationships, we 236 conducted untargeted liquid chromatography-tandem mass spectrometry (LC-MS/MS) analysis 237 of the saliva samples from the same participants who were first investigated for taxonomic 238 profiling in the above analyses [48,49]. By integrating feature based molecular networking 239 [50] with an automated chemical classification [51] and reference frame based differential 
240 abundance analysis [52] approaches, we revealed differential representation of the key molecular

241 features in obesity and depressive symptom conditions.

242 Feature based mass spectral molecular networking (FBMN) and chemically-informed 243 comparison of metabolomic profiles

244 A data matrix of MS1 features that triggered MS2 scans were uploaded along with the metadata 245 file to Global Natural Product Social Molecular Networking (GNPS) (https://gnps.ucsd.edu) 246 [49]. Feature-based molecular networking (version release_20) [50] was performed, and library 247 IDs were generated (see Supplementary Materials and Methods section). To further gain a broad 248 overview of the chemistry of salivary metabolomes from MS/MS data, utilizing an automated 249 chemical classification approach [51], available via GNPS platform, we performed a chemically250 informed comparison of untargeted metabolomic profiles across the four groups.

\section{Differential ranking of taxa and metabolomic features}

252 Differential ranks of taxa and metabolomic features were calculated using Songbird [52], which 253 uses reference frames. Age, sex, race and time of day of saliva collection were provided as 254 covariates in generating a multinomial regression model based on microbial features. 255 Differential microbial features were visualized alongside de novo phylogenetic tree constructed 256 from the representative sequences of amplicon sequence variants (ASVs) obtained in this study 257 using EMPress [23]. Statistical significance was tested by applying LMMs on log-ratios of the 258 top-and bottom-20 ranked microbes for each group obtained using Qurro rank plots [53]. We 259 applied a linear regression model by utilizing log-ratios of bacterial features and BARIC 
260 inflammatory scores to test interactions between obesity-depressive symptoms and inflammation 261 relationships.

262 To mitigate the inter-batch effect often observed in the metabolomics data due to

263 technical limitations in the number of samples processed in a batch, relative abundances were 264 adjusted for batch specific-effect along with age, sex, race and time of day, utilizing the 265 multivariate model in the reference frame-based approach [52]. We chose cluster 1 (90 features) 266 as the denominator ("reference frame") for the log-ratio calculations due to its high prevalence 267 across samples, and moreover, GNPS analyses groups structurally similar molecules into a 268 cluster. Statistical significance was tested by applying Friedman test to account for repeated 269 measurements, prior to multiple pairwise comparison analysis using Wilcoxon rank-sum tests.

270

\section{Microbe-metabolite interactions through their co-occurrence probabilities}

272 Permutation based differential abundance testing was performed using discrete false-discovery 273 rate correction method [54] in Calour (https://github.com/biocore/calour) to remove batch274 specific MS1 molecular features. Annotated features that were not identified as batch-specific 275 were included in the co-occurrence analysis. Using ASV ( $\mathrm{N}=1516)$ and annotated molecular 276 features $(\mathrm{N}=155)$ as inputs to train neural networks [55] in QIIME 2 [56], we estimated the 277 conditional probability that each molecule is present given the presence of a specific 278 microorganism. The resulting conditional probability matrix representing microbe-metabolite 279 interactions was visualized as an EMPeror biplot [55].

\section{Results}




\section{Participant characteristics}

282 A total of 261 saliva samples collected from five time points across the day from 60 participants 283 were analyzed ( 20 - 65 years): 50 participants had five; 51 had four, and 54 had three samples 284 which were adjusted in analyses (See Statistical Analyses). Participants were categorized into the 285 following four groups: non-obese $(\mathrm{BMI}<30 \mathrm{~kg} / \mathrm{m} 2)$ and lower-depressive controls $(\mathrm{N}=10$ 286 participants; $\mathrm{n}=43$ saliva samples; "controls"), obese (BMI $\geq 30 \mathrm{~kg} / \mathrm{m} 2)$ and lower-depressive 287 (N=18; n=74; “Ob/lower-Dep"), non-obese and higher-depressive symptoms (N=5; n=22; "Non288 ob/higher-Dep"), and obese and higher-depressive symptoms (N=27; n=122; “Ob/higher-Dep”).

289 Sociodemographic characteristics are presented across participant groups (Table 1).

\section{Obesity is associated with depressive symptomatology and inflammation}

291 Given that individuals with a clinical diagnosis of depression and/or use of antidepressants were 292 excluded from the study to focus on inflammation-related subclinical depressive symptoms in 293 relation to obesity among otherwise healthy adults, BDI total scores (BDI-T) on average were 294 low (median=3; sd=5; range=0-22). The median value of BDI-T of $\geq 3$ was used to divide 295 participants with relatively 'higher' or 'lower' depressive symptoms in this non-clinical sample. In all individuals, BMI was positively correlated with BDI-T scores $(r=0.29, p=0.04)$, as

297 well as cognitive-affective $(r=0.27, p=0.03)$ and somatic symptom scores with small to medium 298 effects $(r=0.22, p=0.08)$ (Figure S1). BARIC values, an indicator of neuro-inflammation 299 regulation, were negatively correlated with BMI $(r=-0.38, p=0.009)$, and an estimation of 300 adipose tissue volume indicated by \%trunk fat $(r=-0.25, p=0.034)$ across all participants (Figure 301 S1). Age did not moderate any of these relationships, which is in agreement with previous 
302 findings [20]. Altogether, obesity was significantly associated with both inflammation regulation

303 and depressive symptoms. However, no significant associations were observed between BARIC

304 and BDI scores in this study (Figure S1).

305 Oral microbiota differ based on obesity-depressive symptom groups and inflammation

306 status

307 Principal coordinates analysis ( $\mathrm{PCoA})$ and post-hoc pairwise comparisons of unweighted-

308 UniFrac distances of samples revealed that oral microbiota composition was distinct by obesity

309 (PERMANOVA pseudo-F=0.004, $p=0.001$, Figure 1A, Table 2), BDI-T (PERMANOVA

310 pseudo- $\mathrm{F}=0.001, p=0.0$, Figure $1 \mathrm{~B}$, Table 2 ) and across the four obesity-depressive symptom

311 comorbid groups (i.e, Ctrl, Ob/lower-Dep, Non-ob/higher-Dep, Ob/higher-Dep) (Figure 1C,

312 Table 2 and Table 3). Beta-diversity was also significantly differentiated based on the host

313 inflammation across all participants (PERMANOVA pseudo-F $=4.71, p<0.001$, Figure 1D and

314 Table 2). Significant beta-diversity differences were also observed by age, sex, and race but not

315 by sampling time of day (Table 2). Phylogenetic alpha-diversity increased with inflammation

316 (Faith's PD: $\mathrm{t}=-2.312, p=0.025)$. Inflammation had slightly larger effects $\left(\mathrm{R}^{2}=0.02\right)$ on

317 microbiome composition than obesity $\left(\mathrm{R}^{2}=0.008\right)$ and depressive symptomatology $\left(\mathrm{R}^{2}=0.01\right)$

318 (Table 2).

\section{Oral microbiota is predictive of the host obesity-depressive symptomatology}

320 To assess the predictive capacity of the oral microbiome in stratifying individuals with

321 depressive symptoms, obesity and depressive symptomatology-obesity co-occurrence status, we 
322 utilized supervised random forest classification. The prediction performance of the model

323 indicated by both area under the receiver operating characteristic curve (AUROC) and area under

324 precision recall curve (AUPRC), revealed high prediction accuracy (AUROC $=0.75$ and

325 AUPRC $=0.74)$ for obesity-depressive symptom status (Ob/higher Dep) than other groups when

326 multiple samples per-participant were taken into account (Figure 2A and 2B). The Ctrl group

327 was predicted with $\mathrm{AUROC}=0.75$ and $\mathrm{AUPRC}=0.58 ; \mathrm{Ob} /$ lower Dep status with $\mathrm{AUROC}=0.70$

328 and $\mathrm{AUPRC}=0.49$; Non-ob/higher Dep with $\mathrm{AUROC}=0.70$ and $\mathrm{AUPRC}=0.46$. However, at

329 sample-level both AUROC and AUPRC ranged from 0.93 to 0.97, across all groups (Figure S2A

330 and S2B). Altogether, oral microbiome was highly predictive of depressive symptomatology-

331 obesity co-occurrences than obesity and depressive symptomatology independently.

\section{Key oral bacterial taxa are associated with specific host phenotype}

333 Next, we identified the most differentially ranked microbes (99 unique taxa) associated with host 334 phenotypes (Figure 2C). Linear mixed-effects model revealed significant differences in the 335 relative abundances of microbes associated with Ob/higher-Dep ( $\mathrm{t}=6.5, \quad p=5.07 \mathrm{e}-08)$,

336 Non-ob/higher-Dep ( $\mathrm{t}=-4.2, p=0.0002)$ and Ob/lower Dep $(\mathrm{t}=-4.5, p=5.07 \mathrm{e}-05)$ in comparison to

337 Ctrl group, and with inflammation status $(\mathrm{t}=-4.83, p=3.03 \mathrm{e}-05)$. Most differentially represented 338 taxa (84 unique taxa) were assigned to Gram-negative bacteria such as Prevotella, 339 Aggregatibacter, Pseudomonas, Campylobacter, Clostridia (Selenomonas, Butyrivibrio, 340 Veillonella, Megasphaera and Schwartzia), Leptotrichia, Capnocytophaga, and periodontal 341 pathogens such as Treponema, Veillonella, Porphyromonas and Fusobacterium. Gram-positive 342 (15 unique taxa) were assigned to Peptostreptococcaceae, Clostridia (Catonella, 
343 Mogibacteriaceae), Staphylococcus, Corynebacterium, Rothia, Actinomyces, and 344 beneficial/probiotic genera Bifidobacterium and Lactobacillus (Figure 2C, log-fold change

345 abundances for each microbe are shown in Table S1). The Ob/higher-Dep group exhibited a 346 slightly higher abundance of Gram-negative bacteria relative to Gram-positive compared to the 347 Ctrl group (Wilcoxon test: $p=0.004$ ) (Figure 2D), which were not significantly associated with 348 BARIC scores (data not shown).

349 Small molecules detected in saliva are associated with obesity-depressive symptom350 inflammation relationships

351 Untargeted LC-MS/MS analysis of the saliva samples was performed to examine the metabolic

352 potential in the oral ecosystem and understand the intimate link between salivary microbiota and 353 metabolome in obesity-depressive symptom relationships.

354 The most predominant chemical classes identified from automated chemical 355 classification [51] of our samples via GNPS [49] platform were terpenoids, indoles, 356 carbohydrates and carbohydrate conjugates, amino acids, peptides, derivatives of purines and 357 pyrimidines, eicosanoids and linoleic acids (Figure S3). Particularly, molecular structures of 358 diazines, benzotraizoles, imidazopyrimidines and azides were batch-specific (Figure S3). 359 Feature-based mass spectral molecular networking of 7,818 total MS1 molecular features (which 360 included retention time and relative quantitative information) enabled the annotation of 248 that 361 had matches against all publicly available reference spectra [57]. It should be noted that these are

362 level 2 or 3 annotations according to the 2007 metabolomics standards initiative [58]. A 363 reference-frame based approach enabled the identification of 155 features distinctly associated 
364 with specific categories relative to Ctrl group (i.e., Non-Ob/lower-dep) (Figure 3). Key

365 molecules involved in host-microbiota interactions such as the annotation as tyrosine (level 2), a

366 precursor of catecholamine, dopamine and serotonin, and tryptophan (level 2, cluster 14 and 26

367 in Figure 3), a precursor of the neurotransmitter serotonin, were depleted in Ob/higher-Dep and

368 Ob/lower-Dep groups (Figure 2B). The amino acid, phenylalanine (Level 2, cluster 2 Figure 3), a

369 biosynthetic precursor of tyrosine, catecholamine, dopa and dopamine was less abundant in the

$370 \mathrm{Ob} /$ higher-Dep and Non-ob/higher-Dep groups, but increased with inflammation status (Figure $3714 A)$.

372 Within the molecular network, we also identified 41 molecular clusters primarily 373 associated with quorum sensing molecules of microbiota, products of microbial transformation 374 of dietary components or host molecules, and essential aromatic amino acids (Figure 3). Most 375 intriguingly, we identified 34 structurally distinct dipeptides across groups, making it the most 376 prevalent molecular cluster within the network (molecular features of clusters 2, 3, 5, 9, 12, 17, 377 19, 30, 31, 32 and 34 in Figure 3). Of these, molecular features of cluster 2 (present in 60 378 participants) were differentially represented in Ob/higher-Dep and Non-ob/higher-Dep 379 individuals, while features of cluster 34 (present in 58 participants) were differentially 380 represented in Ob/higher-Dep and Ob/lower Dep individuals, when compared to controls (see 381 left panels in Figure 4A). Moreover, clusters 2, 14 and 26 were depleted in the Ob/higher-Dep 382 and non-ob/higher-Dep groups, while cluster 34 was depleted in the Ob/higher-Dep and $383 \mathrm{Ob} /$ lower Dep groups. Other differentially represented molecular clusters included clusters 14 384 (detected in 56 participants) and 26 (detected in 58 participants), which encompassed two of the 385 essential aromatic amino acids i.e. tryptophan and tyrosine molecules (see clusters 14 and 26 in 
386 Figure 3, Figure 4A). Molecular features from these clusters are positively associated with 387 inflammation (right panels in Figure 4A). Abundance of features from the remaining clusters did 388 not significantly vary across groups (data not shown). Other molecular features included 389 previously reported microbiota-derived dipeptides (Phe-Val and Tyr-Val) (see clusters 2 and 30 390 in Figure 3) [42,59,60]. Dipeptide (Phe-Phe) reported to be synthesized by Clostridium (cluster 2 391 Figure 3) [61] was predominant in the Ob/higher-Dep group. Other molecules such cyclic 392 dipeptides (Val-Pro and Val-Leu), commonly found to be made by microbes, were also 393 identified (see cluster 2 and 12 Figure 3, Figure 4A, Table S2) $[59,60]$. The majority of the other 394 dipeptides identified were potentially related to host dietary metabolism (i.e. enzymatic digest of 395 food proteins) [43,44]. Among these, Tyr-Leu, Phe-Leu and Ile-Tyr (cluster 2 Figure 3), were 396 significantly more abundant in the Ctrl group compared to the other Ob/higher-Dep and 397 Ob/lower-Dep groups (Figure 4A) among which, Tyr-Pro (cluster 34 Figure 3) was also depleted 398 (Figure 4A).

399 Key oral microbes co-occurred with biosynthetic precursors of the neurotransmitters and 400 dipeptide signaling molecules

401 Integration of the microbiome and metabolomics data revealed associations between oral 402 microbial metabolism and key oral microbes such as Prevotella, Clostridia, Selenomonas, 403 Aggregatibacter, Oribacterium, Corynebacterium, and periodontal pathogens such as Tannerella 404 and Porphyromonas (Figure 4B). Dipeptide signaling molecules (Phe-Phe, Phe-Val and Tyr-Val) 405 co-occurred with Clostridia, Prevotella and Porphyromonas, corroborating known associations 406 of dipeptides produced by Clostridium spp. [42,59-61]. Members of Clostridia also co-occurred 
407 with phenylalanine, a potential biosynthetic precursor of dopamine, epinephrine and tryptophan.

408 Intriguingly, Oribacterium belonging to Clostridium and Tannerella co-occurred with 409 tryptophan, shown to encompass tryptophan biosynthetic pathways. Our findings further 410 corroborate known microbial-derived cyclic dipeptides (Val-Leu and Val-Pro) associations with

411 Selenomonas, Aggregatibacter and Clostridium spp. (Figure 4B) [59,60]. Potential dietary 412 dipeptides (Phe-Leu, Tyr-Pro and Tyr-Leu) co-occurred with Tannerella, Selenomonas, 413 Prevotella, Porphyromonas and Clostridia $[43,44]$.

\section{Discussion}

415 We previously reported that obesity is significantly associated with both inflammation and 416 depressive symptoms [20,21,47]. Growing evidence also suggests that gut bacterial composition 417 and their specialized metabolites may trigger chronic systemic inflammation in obesity418 depression co-occurrences [2], highlighting the importance of the host immune and microbial 419 interplay. In this study, we showed that the composition of salivary microbiota differ in co420 occurring obesity-depressive symptoms and in relation to obesity, depression, and inflammation.

421 We also showed that individual bacterial taxa were linked to specific host obesity-depressive 422 symptoms 'phenotype', and small-molecule mediated microbe-microbe and microbe-host 423 interactions likely play a critical role in these host phenotypes. While effects of obesity, 424 inflammation and depression phenotypes on gut microbiome have been studied previously, this 425 study extends our previous work [33] that identified relationships between oral microbial 426 composition, host stress profile and inflammatory status, by providing further evidence that oral 427 microbial composition and metabolic profiles are also influenced by the specific host 
428 phenotypes, and are likely characterized by significant alterations in the biosynthetic precursors

429 of neurotransmitters and signaling dipeptides. These findings highlight a potential link between

430 oral microbiota and the brain (i.e. oral-brain axis), adding to known gut microbiota-brain

431 interactions [34-36], as well as biomarker utility of oral microbiome in studying brain and

432 behavioral outcomes.

433 Examining the composition of the oral microbiome revealed significant differences based

434 on obesity, depressive symptomatology and comorbid obesity-depressive symptomatology. At

435 the same time, the oral microbiome composition differed by the host inflammatory processes

436 beyond the effects of obesity or depression. This emphasizes the need of further scrutinizing the

437 central role of microbiome-mediated inflammation in obesity-depressive symptomatology

438 interrelationship and is closely aligned with the existing literature in chronic low-grade

439 inflammation at the intersection of depression and obesity.

440 Random forest classification indicated that oral microbiota is highly predictive of 441 obesity-depressive symptom co-occurrences, suggesting specific microbial signatures associated

442 with obesity-depression co-occurrences. Corroborating these findings, abundances of several 443 microbes were differentially represented across the obesity-depressive symptomatology groups 444 as revealed by the differential abundance analysis. Gram-negative microbes have been shown to 445 be associated with inflammation due to their LPS cell wall, the hallmark trait of Gram-negative 446 bacteria. We found that Gram-negative microbes Prevotella, Aggregatibacter, Pseudomonas, 447 Campylobacter, Selenomonas, Leptotrichia, Capnocytophaga, and Gram-negative periodontal 448 pathogens such as Treponema, Veillonella, Porphyromonas and Fusobacterium are enriched in $449 \mathrm{Ob} /$ higher-dep group. However, we found no significant correlation with BARIC scores that 
measured monocytes' responsiveness to a $\beta$-AR agonist during an inflammatory response to LPS,

451 indicating inflammation regulatory processes [47]. Increased abundance of Prevotella in the

452 human oral cavity has been previously ambiguously associated with both health and disease

453 conditions [26,62,63]. Pathogenic Campylobacter has been shown to increase anxiety-like

454 behavior in mice [64] and Aggregatibacter has been reported to be associated with inflammation.

455 Notably, Gram-positive beneficial microbes Bifidobacterium and Lactobacillus depleted in

$456 \mathrm{Ob} /$ higher-Dep group are in line with their activity as they are reported to exhibit antidepressant

457 and anti-obesity effects, and reduced levels of TNF- $\alpha$ in both clinical and animal studies [65-67].

458 All of these differentially abundant oral taxa present potential biomarkers in obesity-depression

459 co-occurrences, however, more studies are needed to further confirm these findings, as our study

460 did not find significant differences in the abundances of microbes at genera-level.

461 We also found differences in relative abundance patterns in many molecules across the

462 obesity-depression symptoms groups, including quorum sensing molecules of microbiota,

463 products of microbial transformation of dietary components or host molecules and aromatic

464 amino acids. Importantly, metabolites of aromatic amino acids tryptophan and tyrosine, both of

465 which are precursors of the neurotransmitter serotonin, have been mechanistically implicated in

466 obesity-depression associations [68], and play signaling roles in host-microbe interactions in the

467 gut [69], were depleted in obese individuals compared to the control group. Host dietary

468 dipeptides (Tyr-Leu and Phe-Leu) that were significantly less abundant among the obese

469 individuals compared to the control group in this study are shown to display anti-depressant-like

470 activity as greater abundance of Tyr-Leu activates serotonin, dopamine and gamma aminobutyric

471 acid (GABA) receptors in mice $[43,44]$. Tyr-Pro and Ile-Tyr, which were also depleted in the 
472 obese individuals in our study, are an inhibitor of angiotensin I-converting enzyme (ACE) with 473 antihypertensive activity [70] and affect catecholamine (e.g. dopamine and noradrenaline) 474 metabolism in the mouse brain [71], respectively. These findings offer initial mechanistic insight 475 into comorbid obesity and depression, albeit complex.

476 Furthermore, we identified several structurally distinct dipeptides that were positively 477 associated with inflammation. To our knowledge, it is the first time that microbial-derived 478 dipeptide (Phe-Val, Tyr-Val and Phe-Phe) and cyclic dipeptides signaling molecules (Val-Pro 479 and Val-Leu) were detected in salivary metabolomes. Biosynthetic gene clusters and the 480 production of dipeptides (Phe-Val and Tyr-Val) have been recently identified in the human 481 microbiome $[42,59,60]$. These molecules are known to play key roles in quorum sensing (cell-to482 cell communication to maintain cell density) and virulence, and promote growth of beneficial 483 Bifidobacterium [41]. A previous study showed that Phe-Phe derived from Clostridium sp. can 484 inhibit host proteins by chemical modification of the host cellular proteins, especially by 485 targeting cathepsins in human cell proteomes [61]. Given our findings that Phe-Phe was highly 486 abundant in the Ob/higher-Dep group, its biological role in the cellular inflammatory process 487 which likely underlie obesity-depression comorbidity warrants further investigation.

488 Our findings of specific microbe-metabolite interactions with potential to influence host's 489 brain functioning offer potentially significant insight into the role of host immune-microbiome 490 interplay in comorbid obesity-depression and is likely through microbial neurotransmitters. 491 Metabolic pathways for biosynthesis of neuroactive molecules in the genomes of human492 associated genera Clostridium and Tannerella have been recently reported [35]. Intriguingly, 493 members of Clostridium and Tannerella co-occurred with tryptophan and have been 
494 detected/reported to harbor genes for tryptophan biosynthesis [35]. Members of Clostridia co-

495 occurred with phenylalanine, a potential biosynthetic precursor of dopamine, epinephrine and

496 tryptophan, have been shown to be key species in neuropsychiatric disorders and shown to

497 produce dopamine in mice [36,72]. Many of these molecules including the dipeptides, shown to

498 have potential to cross the intestinal barrier and blood brain barrier, may modulate the oral-brain

499 connection through neurotransmitter signaling pathways [35,72]. Such neurotransmitters and

500 their biosynthetic precursors may offer promising targets for therapeutics.

501 There is a caveat in this study that merits caution: in an effort to recruit individuals with

502 subclinical levels of depressive mood co-occurring with a range of obesity without

503 antidepressant intake or heterogeneous clinical depression, the participants exhibited low levels

504 of BDI scores on average which may limit the applicability of our findings to clinical depression.

505 At the same time, it is notable that host-microbiome-metabolome signatures and their

506 interactions appear to be salient in pathophysiology of subclinical depression symptomatology.

507 We also acknowledge a small sample size of the study participants, in spite of the expanded

508 specimen sample size owing to multiple saliva collections.

\section{Conclusions}

510 Despite these limitations, our study significantly expands the evidence for microbial specialized

511 metabolites and peptides with neuroactive potential, adding further research avenues into

512 microbiome-host physiology interactions and there is a great deal of clinical potential in

513 understanding and modifying these interactions. Furthermore, it provides initial evidence for a 
514 foundation of the microbial oral-brain axis in addition to the gut-brain axis in the context of

515 obesity-depression-inflammation interrelationships.

\section{Declarations}

\section{Ethics approval and consent to participate}

518 All participants provided informed consent to the protocol prior to the commencement of the

519 study. The Ethics Committee of the University of California, San Diego, CA, USA, approved the 520 study design as well as the procedure for obtaining informed consent (IRB reference number:

521 171027). All experiments were performed in accordance with the approved guidelines of UCSD

522 Human Research Protections Program.

\section{Consent for publication}

524 Not applicable

\section{Availability of data and material}

526 Sample metadata, the raw and processed $16 \mathrm{~S}$ sequencing data and their associated feature tables,

527 and preparation metadata are available in Qiita Study ID 11259

528 (https://qiita.ucsd.edu/study/description/11259). Mass spectral files and LC-MS/MS preparation

529 metadata are accessible from the MassIVE repository accession ID MSV000083077

530 (ftp://massive.ucsd.edu/MSV000083077). The GNPS feature based molecular networking job is 
531 available

532 task=f192a0030f694224a0ba8f08223a1323

\section{Competing interests}

534 PCD serves as a scientific advisor to Sirenas, Cybele and Galileo. PCD is also a founder and 535 scientific advisor of Ometa and Enveda with approval by UC San Diego.

\section{Funding}

537 This work was supported in part by R01 HL90975 and 90975S1 from the NIH (Hong), a Seed

538 Grant from the Center for Microbiome Innovation at UC San Diego (Hong), Wayne State 539 University Endowment Fund (Hong), and the Kavli Institute for Brain and Mind (KIBM) 540 Innovative Research Grant (Aleti).

\section{Authors' contributions}

542 Hong designed and obtained funding for the study. GA performed the data analysis. JNK, KW,

543 AT, ADS and Huang assisted with the data analysis. GA, Hong, PCD, ADS and RK interpreted 544 the results. GA, ET and Hong wrote the original manuscript. GA, JNK, ET, KW, AT, Huang, 545 ADS, Hong, PCD and RK reviewed and edited the manuscript.

\section{Acknowledgements}

547 Not applicable 
548

549

550

551

552

553

554

555

556

557

558

559

560

561

562

563

564

565

566

567

568

569

570

571

572

573

574

575

576

577 11. Milano W, Ambrosio P, Carizzone F, De Biasio V, Di Munzio W, Foia MG, et al. 578 Depression and Obesity: Analysis of Common Biomarkers. Diseases. MDPI AG; 2020;8:23.

\section{References}

1. Smith DJ, Court H, McLean G, Martin D, Martin JL, Guthrie B, et al. Depression and multimorbidity: A cross-sectional study of 1,751,841 patients in primary care. J Clin Psychiatry. Physicians Postgraduate Press Inc.; 2014;75:1202-8.

2. Schachter J, Martel J, Lin CS, Chang CJ, Wu TR, Lu CC, et al. Effects of obesity on depression: A role for inflammation and the gut microbiota. Brain. Behav. Immun. Academic Press Inc.; 2018. p. 1-8.

3. James SL, Abate D, Abate KH, Abay SM, Abbafati C, Abbasi N, et al. Global, regional, and national incidence, prevalence, and years lived with disability for 354 Diseases and Injuries for 195 countries and territories, 1990-2017: A systematic analysis for the Global Burden of Disease Study 2017. Lancet. Lancet Publishing Group; 2018;392:1789-858.

4. Mannan M, Mamun A, Doi S, Clavarino A. Is there a bi-directional relationship between depression and obesity among adult men and women? Systematic review and bias-adjusted meta analysis. Asian J. Psychiatr. Elsevier B.V.; 2016. p. 51-66.

5. Dawes AJ, Maggard-Gibbons M, Maher AR, Booth MJ, Miake-Lye I, Beroes JM, et al. Mental health conditions among patients seeking and undergoing bariatric surgery a metaanalysis. JAMA - J Am Med Assoc. American Medical Association; 2016;315:150-63.

6. Pratt LA, Brody DJ. Depression and obesity in the U.S. adult household population, 20052010. NCHS Data Brief. NCHS Data Brief; 2014;1-8.

7. Luppino FS, De Wit LM, Bouvy PF, Stijnen T, Cuijpers P, Penninx BWJH, et al. Overweight, obesity, and depression: A systematic review and meta-analysis of longitudinal studies. Arch. Gen. Psychiatry. Arch Gen Psychiatry; 2010. p. 220-9.

8. Woo YS, Seo HJ, McIntyre RS, Bahk WM. Obesity and its potential effects on antidepressant treatment outcomes in patients with depressive disorders: A literature review. Int. J. Mol. Sci. MDPI AG; 2016.

74 9. Scully T. Public health: Society at large. Nature. Nature Publishing Group; 2014;508:S50-1.

75 10. Capuron L, Lasselin J, Castanon N. Role of Adiposity-Driven Inflammation in Depressive Morbidity. Neuropsychopharmacology. Nature Publishing Group; 2017. p. 115-28. 
579 12. Young JJ, Bruno D, Pomara N. A review of the relationship between proinflammatory 580 cytokines and major depressive disorder. J. Affect. Disord. Elsevier B.V.; 2014. p. 15-20.

581 13. Ouchi N, Parker JL, Lugus JJ, Walsh K. Adipokines in inflammation and metabolic disease. 582 Nat. Rev. Immunol. Nat Rev Immunol; 2011. p. 85-97.

583 14. Dalmas E, Clément K, Guerre-Millo M. Defining macrophage phenotype and function in 584 adipose tissue. Trends Immunol. Trends Immunol; 2011. p. 307-14.

585 15. Wohleb ES, McKim DB, Sheridan JF, Godbout JP. Monocyte trafficking to the brain with 586 stress and inflammation: A novel axis of immune-to-brain communication that influences mood 587 and behavior. Front. Neurosci. Frontiers Media S.A.; 2015.

588 16. Miller AH, Raison CL. The role of inflammation in depression: From evolutionary 589 imperative to modern treatment target. Nat. Rev. Immunol. Nature Publishing Group; 2016. p. $59022-34$.

591 17. Osimo EF, Pillinger T, Rodriguez IM, Khandaker GM, Pariante CM, Howes OD. 592 Inflammatory markers in depression: A meta-analysis of mean differences and variability in 593 5,166 patients and 5,083 controls. Brain. Behav. Immun. Academic Press Inc.; 2020. p. 901-9.

594 18. Milaneschi Y, Lamers F, Berk M, Penninx BWJH. Depression Heterogeneity and Its 595 Biological Underpinnings: Toward Immunometabolic Depression. Biol. Psychiatry. Elsevier 596 USA; 2020. p. 369-80.

597 19. Hong S. Inflammation at the interface of physical and neuropsychiatric outcomes: 598 Investigation of neuroendocrine regulatory pathways to inform therapeutics. Brain. Behav. 599 Immun. Academic Press Inc.; 2020. p. 270-4.

600 20. Kohn JN, Cabrera Y, Dimitrov S, Guay-Ross N, Pruitt C, Shaikh FD, et al. Sex-specific roles 601 of cellular inflammation and cardiometabolism in obesity-associated depressive 602 symptomatology. Int J Obes. Nature Publishing Group; 2019;43:2045-56.

603 21. Cheng T, Dimitrov S, Pruitt C, Hong S. Glucocorticoid mediated regulation of inflammation 604 in human monocytes is associated with depressive mood and obesity. 605 Psychoneuroendocrinology. Elsevier Ltd; 2016;66:195-204.

606 22. Sharma S, Fulton S. Diet-induced obesity promotes depressive-like behaviour that is 607 associated with neural adaptations in brain reward circuitry. Int J Obes. Int J Obes (Lond); 608 2013;37:382-9.

609 23. Schneeberger M, Everard A, Gómez-Valadés AG, Matamoros S, Ramírez S, Delzenne NM, 610 et al. Akkermansia muciniphila inversely correlates with the onset of inflammation, altered 
611 adipose tissue metabolism and metabolic disorders during obesity in mice. Sci Rep. Nature

612 Publishing Group; 2015;5.

613 24. Cani PD, Amar J, Iglesias MA, Poggi M, Knauf C, Bastelica D, et al. Metabolic endotoxemia 614 initiates obesity and insulin resistance. Diabetes. Diabetes; 2007;56:1761-72.

615 25. Chen T, Yu WH, Izard J, Baranova O V., Lakshmanan A, Dewhirst FE. The Human Oral 616 Microbiome Database: a web accessible resource for investigating oral microbe taxonomic and 617 genomic information. Database (Oxford). Database (Oxford); 2010;2010.

618 26. Dewhirst FE, Chen T, Izard J, Paster BJ, Tanner ACR, Yu WH, et al. The human oral 619 microbiome. J Bacteriol. J Bacteriol; 2010;192:5002-17.

620 27. Schmidt TSB, Hayward MR, Coelho LP, Li SS, Costea PI, Voigt AY, et al. Extensive 621 transmission of microbes along the gastrointestinal tract. Elife. eLife Sciences Publications Ltd; $622 \quad 2019 ; 8$.

623 28. Atarashi K, Suda W, Luo C, Kawaguchi T, Motoo I, Narushima S, et al. Ectopic colonization 624 of oral bacteria in the intestine drives TH1 cell induction and inflammation. Science (80- ). 625 American Association for the Advancement of Science; 2017;358:359-65.

626 29. Dickson I. Gut microbiota: Oral bacteria: A cause of IBD? Nat. Rev. Gastroenterol. Hepatol. 627 Nature Publishing Group; 2018. p. 4-5.

628 30. Dominy SS, Lynch C, Ermini F, Benedyk M, Marczyk A, Konradi A, et al. Porphyromonas 629 gingivalis in Alzheimer's disease brains: Evidence for disease causation and treatment with 630 small-molecule inhibitors. Sci Adv. American Association for the Advancement of Science; $6312019 ; 5$.

632 31. Farrokhi V, Nemati R, Nichols FC, Yao X, Anstadt E, Fujiwara M, et al. Bacterial 633 lipodipeptide, Lipid 654, is a microbiome-associated biomarker for multiple sclerosis. Clin 634 Transl Immunol. John Wiley and Sons Inc; 2013;2.

635 32. Shen L. Gut, oral and nasal microbiota and Parkinson's disease. Microb. Cell Fact. BioMed 636 Central Ltd.; 2020.

637 33. Kohn JN, Kosciolek T, Marotz C, Aleti G, Guay-Ross RN, Hong SH, et al. Differing salivary 638 microbiome diversity, community and diurnal rhythmicity in association with affective state and 639 peripheral inflammation in adults. Brain Behav Immun. Academic Press Inc.; 2020;87:591-602.

640 34. Yano JM, Yu K, Donaldson GP, Shastri GG, Ann P, Ma L, et al. Indigenous bacteria from 641 the gut microbiota regulate host serotonin biosynthesis. Cell. Cell Press; 2015;161:264-76. 
642 35. Valles-Colomer M, Falony G, Darzi Y, Tigchelaar EF, Wang J, Tito RY, et al. The 643 neuroactive potential of the human gut microbiota in quality of life and depression. Nat 644 Microbiol. Nature Publishing Group; 2019;4:623-32.

645 36. Olsen I, Hicks SD. Oral microbiota and autism spectrum disorder (ASD). J. Oral Microbiol. 646 Taylor and Francis Ltd.; 2020.

647 37. Olsen I. Update on bacteraemia related to dental procedures. Transfus Apher Sci. Transfus 648 Apher Sci; 2008;39:173-8.

649 38. Aleti G, Baker JL, Tang X, Alvarez R, Dinis M, Tran NC, et al. Identification of the bacterial 650 biosynthetic gene clusters of the oral microbiome illuminates the unexplored social language of 651 bacteria during health and disease. MBio. American Society for Microbiology; 2019;10:1-19.

652 39. Garg N, Luzzatto-Knaan T, Melnik A V., Caraballo-Rodríguez AM, Floros DJ, Petras D, et 653 al. Natural products as mediators of disease. Nat. Prod. Rep. Royal Society of Chemistry; 2017. 654 p. 194-219.

655 40. Donia MS, Fischbach MA. Small molecules from the human microbiota. Science (80- ). 656 American Association for the Advancement of Science; 2015;349:1254766.

657 41. Hatanaka M, Morita H, Aoyagi Y, Sasaki K, Sasaki D, Kondo A, et al. Effective bifidogenic 658 growth factors cyclo-Val-Leu and cyclo-Val-Ile produced by Bacillus subtilis C-3102 in the 659 human colonic microbiota model. Sci Rep. Nature Research; 2020;10.

660 42. Cao L, Shcherbin E, Mohimani H. A Metabolome- and Metagenome-Wide Association 661 Network Reveals Microbial Natural Products and Microbial Biotransformation Products from the 662 Human Microbiota. mSystems. American Society for Microbiology; 2019;4.

663 43. Mizushige T, Uchida T, Ohinata K. Dipeptide tyrosyl-leucine exhibits antidepressant-like 664 activity in mice. Sci Rep. Nature Research; 2020;10.

665 44. Kanegawa N, Suzuki C, Ohinata K. Dipeptide Tyr-Leu (YL) exhibits anxiolytic-like activity 666 after oral administration via activating serotonin 5-HT1A, dopamine D1 and GABAA receptors 667 in mice. FEBS Lett. FEBS Lett; 2010;584:599-604.

668 45. Franzosa EA, Sirota-Madi A, Avila-Pacheco J, Fornelos N, Haiser HJ, Reinker S, et al. Gut 669 microbiome structure and metabolic activity in inflammatory bowel disease. Nat Microbiol. 670 Nature Publishing Group; 2019;4:293-305.

671 46. Beck AT, Steer RA, Ball R, Ranieri WF. Comparison of Beck depression inventories -IA and 672 -II in psychiatric outpatients. J Pers Assess. J Pers Assess; 1996;67:588-97. 
673 47. Hong S, Dimitrov S, Cheng T, Redwine L, Pruitt C, Mills PJ, et al. Beta-adrenergic receptor

674 mediated inflammation control by monocytes is associated with blood pressure and risk factors

675 for cardiovascular disease. Brain Behav Immun. Academic Press Inc.; 2015;50:31-8.

676 48. Aron AT, Gentry EC, McPhail KL, Nothias LF, Nothias-Esposito M, Bouslimani A, et al.

677 Reproducible molecular networking of untargeted mass spectrometry data using GNPS. Nat

678 Protoc. Nature Research; 2020;15:1954-91.

679 49. Wang M, Carver JJ, Phelan V V., Sanchez LM, Garg N, Peng Y, et al. Sharing and 680 community curation of mass spectrometry data with Global Natural Products Social Molecular 681 Networking. Nat. Biotechnol. Nature Publishing Group; 2016. p. 828-37.

682 50. Nothias LF, Petras D, Schmid R, Dührkop K, Rainer J, Sarvepalli A, et al. Feature-based 683 molecular networking in the GNPS analysis environment. Nat Methods. Nature Research; $6842020 ; 17: 905-8$.

685 51. Tripathi A, Vázquez-Baeza Y, Gauglitz JM, Wang M, Dührkop K, Nothias-Esposito M, et al. 686 Chemically informed analyses of metabolomics mass spectrometry data with Qemistree. Nat 687 Chem Biol. Nature Research; 2021;17:146-51.

688 52. Morton JT, Marotz C, Washburne A, Silverman J, Zaramela LS, Edlund A, et al. 689 Establishing microbial composition measurement standards with reference frames. Nat Commun. 690 Nature Publishing Group; 2019;10.

691 53. Fedarko MW, Martino C, Morton JT, González A, Rahman G, Marotz CA, et al. 692 Visualizing'omic feature rankings and log-ratios using Qurro. NAR Genomics Bioinforma. 693 Oxford University Press (OUP); 2020;2.

694 54. Jiang L, Amir A, Morton JT, Heller R, Arias-Castro E, Knight R. Discrete False-Discovery 695 Rate Improves Identification of Differentially Abundant Microbes. mSystems. American Society 696 for Microbiology; 2017;2.

697 55. Morton JT, Aksenov AA, Nothias LF, Foulds JR, Quinn RA, Badri MH, et al. Learning 698 representations of microbe-metabolite interactions. Nat Methods. Nature Research; $6992019 ; 16: 1306-14$.

700 56. Bolyen E, Rideout JR, Dillon MR, Bokulich NA, Abnet CC, Al-Ghalith GA, et al. 701 Reproducible, interactive, scalable and extensible microbiome data science using QIIME 2. Nat. 702 Biotechnol. Nature Publishing Group; 2019. p. 852-7.

703 57. Aksenov AA, Da Silva R, Knight R, Lopes NP, Dorrestein PC. Global chemical analysis of 704 biology by mass spectrometry. Nat. Rev. Chem. Nature Publishing Group; 2017. p. 1-20. 
705 58. Sumner LW, Amberg A, Barrett D, Beale MH, Beger R, Daykin CA, et al. Proposed 706 minimum reporting standards for chemical analysis: Chemical Analysis Working Group 707 (CAWG) Metabolomics Standards Initiative (MSI). Metabolomics. Metabolomics; 2007;3:21170821.

709 59. Park HB, Crawford JM. Pyrazinone protease inhibitor metabolites from Photorhabdus 710 luminescens. J Antibiot (Tokyo). Nature Publishing Group; 2016;69:616-21.

711 60. Wyatt MA, Mok MCY, Junop M, Magarvey NA. Heterologous Expression and Structural 712 Characterisation of a Pyrazinone Natural Product Assembly Line. ChemBioChem. 713 Chembiochem; 2012;13:2408-15.

714 61. Guo CJ, Chang FY, Wyche TP, Backus KM, Acker TM, Funabashi M, et al. Discovery of 715 Reactive Microbiota-Derived Metabolites that Inhibit Host Proteases. Cell. Cell Press; $716 \quad 2017 ; 168: 517-526 . e 18$.

717 62. Zhang L, Liu Y, Zheng HJ, Zhang CP. The Oral Microbiota May Have Influence on Oral 718 Cancer. Front Cell Infect Microbiol. Frontiers Media S.A.; 2020;9.

719 63. Larsen JM. The immune response to Prevotella bacteria in chronic inflammatory disease. 720 Immunology. Blackwell Publishing Ltd; 2017. p. 363-74.

721 64. Goehler LE, Park SM, Opitz N, Lyte M, Gaykema RPA. Campylobacter jejuni infection 722 increases anxiety-like behavior in the holeboard: Possible anatomical substrates for 723 viscerosensory modulation of exploratory behavior. Brain Behav Immun. Brain Behav Immun; $724 \quad 2008 ; 22: 354-66$.

725 65. Abildgaard A, Elfving B, Hokland M, Lund S, Wegener G. Probiotic treatment protects 726 against the pro-depressant-like effect of high-fat diet in Flinders Sensitive Line rats. Brain Behav 727 Immun. Academic Press Inc.; 2017;65:33-42.

728 66. Abildgaard A, Elfving B, Hokland M, Wegener G, Lund S. Probiotic treatment reduces 729 depressive-like behaviour in rats independently of diet. Psychoneuroendocrinology. Elsevier Ltd; $730 \quad 2017 ; 79: 40-8$.

731 67. Schellekens H, Torres-Fuentes C, van de Wouw M, Long-Smith CM, Mitchell A, Strain C, et 732 al. Bifidobacterium longum counters the effects of obesity: Partial successful translation from 733 rodent to human. EBioMedicine. Elsevier B.V.; 2021;63.

734 68. Chaves Filho AJM, Lima CNC, Vasconcelos SMM, de Lucena DF, Maes M, Macedo D. 735 IDO chronic immune activation and tryptophan metabolic pathway: A potential 
736 pathophysiological link between depression and obesity. Prog. Neuro-Psychopharmacology Biol.

737 Psychiatry. Elsevier Inc.; 2018. p. 234-49.

738 69. Roager HM, Licht TR. Microbial tryptophan catabolites in health and disease. Nat. Commun.

739 Nature Publishing Group; 2018.

740 70. Yamamoto N, Maeno M, Takano T. Purification and Characterization of an Antihypertensive

741 Peptide from a Yogurt-Like Product Fermented by Lactobacillus helveticus CPN4. J Dairy Sci.

742 American Dairy Science Association; 1999;82:1388-93.

743 71. Moriyasu K, Ichinose T, Nakahata A, Tanaka M, Matsui T, Furuya S. The Dipeptides Ile-Tyr 744 and Ser-Tyr Exert Distinct Effects on Catecholamine Metabolism in the Mouse Brainstem. Int J 745 Pept. Hindawi Limited; 2016;2016.

746 72. Asano Y, Hiramoto T, Nishino R, Aiba Y, Kimura T, Yoshihara K, et al. Critical role of gut 747 microbiota in the production of biologically active, free catecholamines in the gut lumen of mice. 748 Am J Physiol - Gastrointest Liver Physiol. Am J Physiol Gastrointest Liver Physiol; 2012;303. 
750 Figure 1. Principal coordinates analyses (PCoA) of oral bacterial communities in (A) non-obese 751 and obese (B) low depressive and higher depressive (C) non-obese low-depressive, non-obese

752 high-depressive, obese, and co-occurring obesity and depressive symptom groups, and (D) in 753 inflammation status. Unweighted-UniFrac distances among samples were visualized using

754 EMPeror. Significance of separation between the groups and further post-hoc pairwise 755 comparisons between groups was tested by applying PERMANOVA test on the principal 756 coordinates.

A

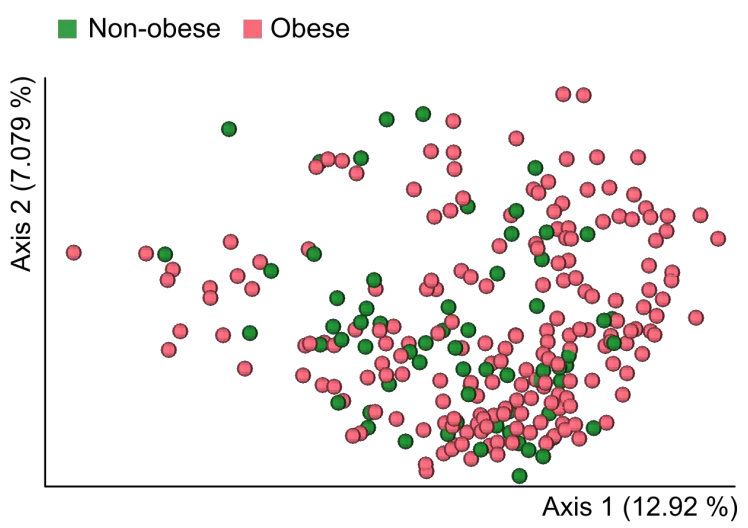

C

Non-obese low-depressive Obese high-depressive

Non-obese high-depressive Obese low-depressive

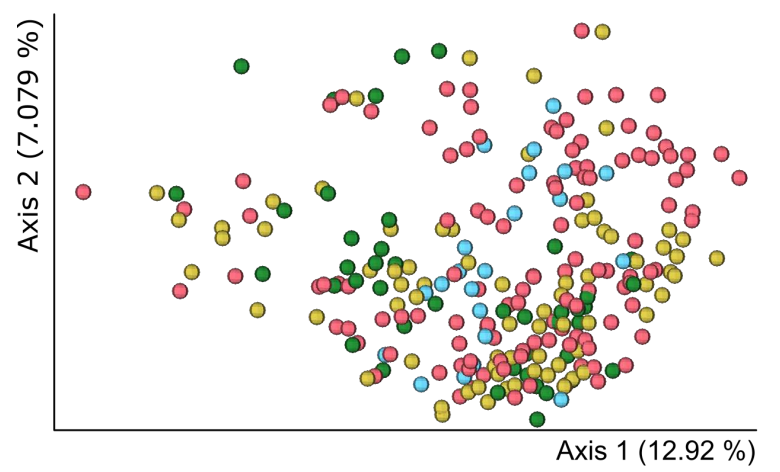

B

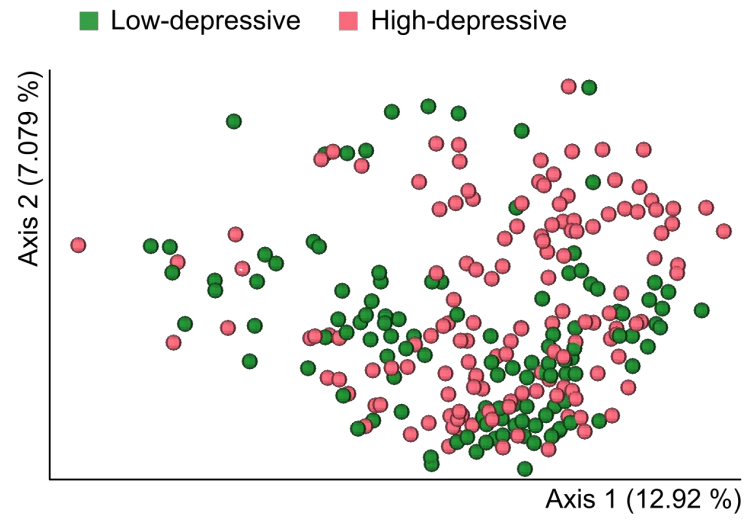

D

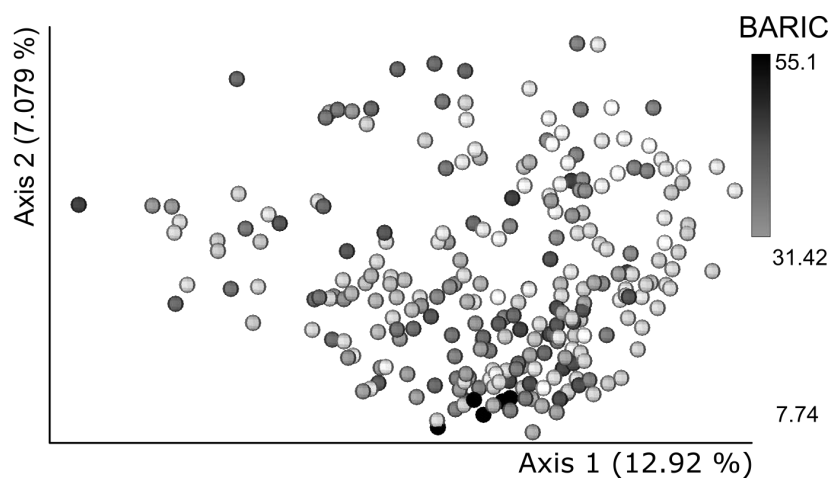


757 Figure 2. Oral microbiota is distinctly impacted by the host status in co-occurring obesity-

758 depressive status. (A) Receiver operating characteristic curves (AUROC) illustrating 759 classification accuracy of the random forest model across all groups (i.e. controls, Ob/lower Dep, 760 Non-ob/higher-Dep, Ob/higher-Dep). (B) Area under precision recall curves (AUPRC) 761 illustrating performance of the random forest model across all groups. (C) Phylogenetic 762 distribution of the most differentially ranked taxa across the groups. Branches of the de novo 763 phylogenetic tree and the innermost ring are colored by phyla. Each barplot layer represents log764 fold change abundances of taxa within the group in comparison to the healthy controls i.e. Non765 ob/lower-Dep. A multinomial regression model was employed for regressing log-fold change 766 abundances against BARIC values. (D) Log-fold change abundances of Gram-negative microbes 767 relative to Gram-positive microbes across host phenotypes. 
A

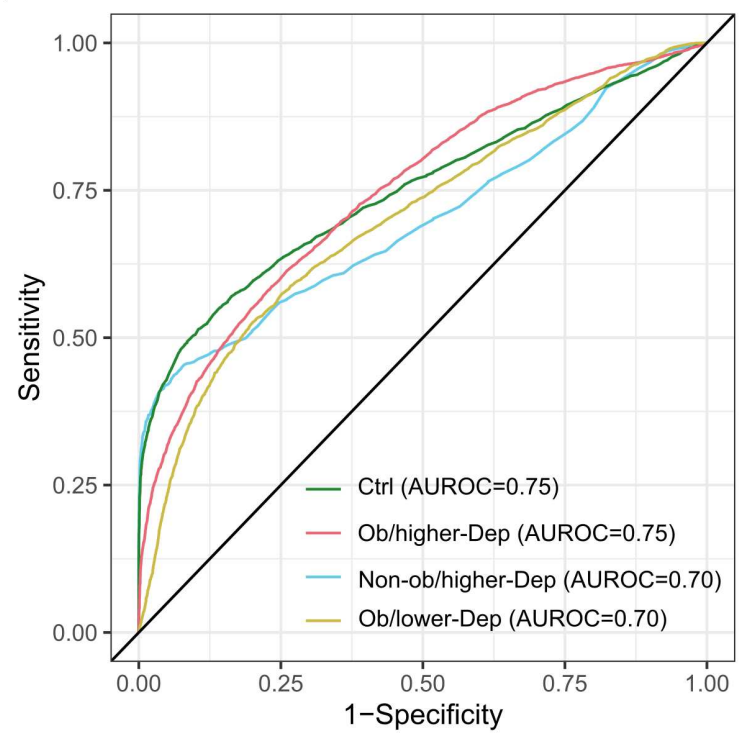

B

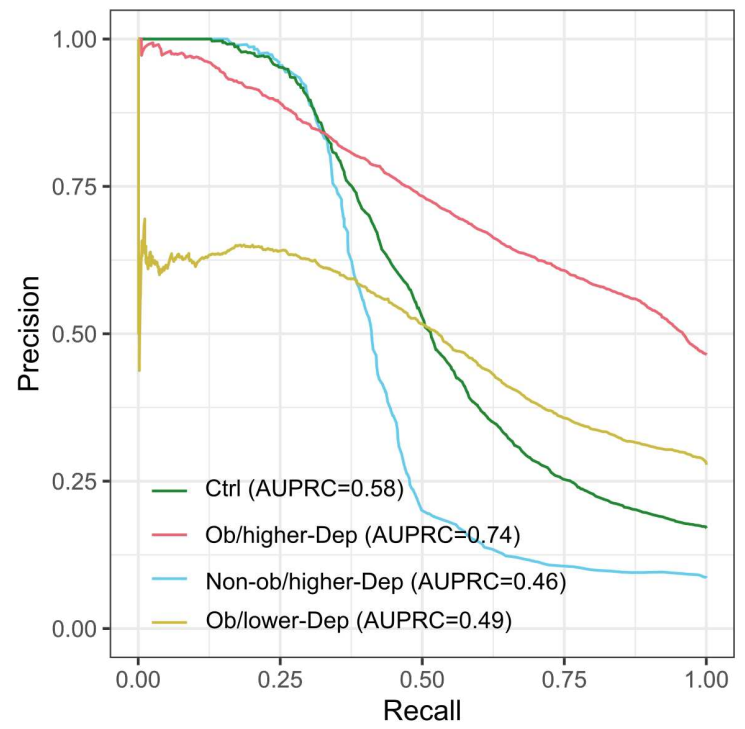

D
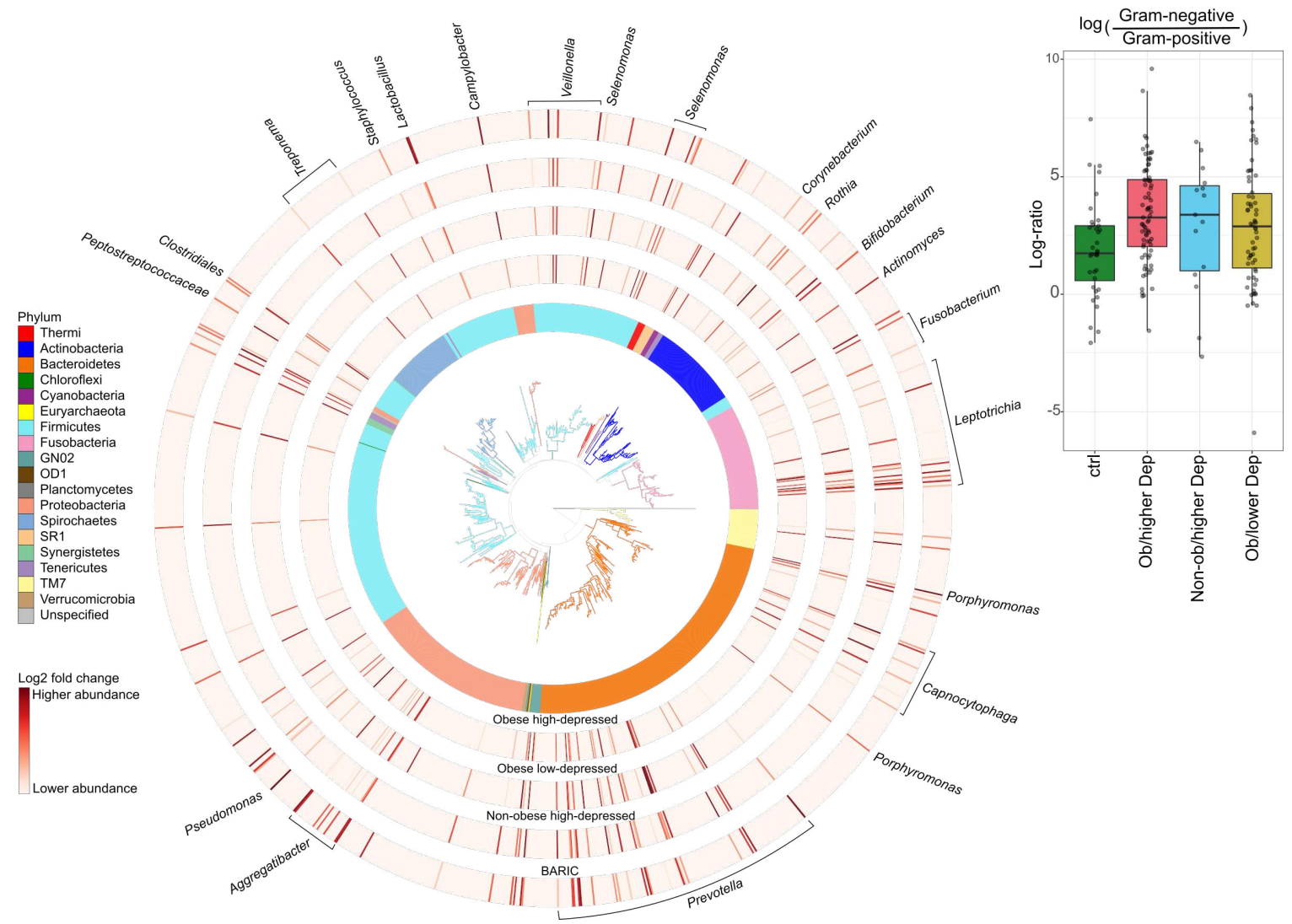

C 
768 Figure 3. Feature-based molecular network of the ions detected in salivary metabolomes of 769 obese-depressive group. The molecular network was generated by 293 nodes with 41 molecular 770 clusters, which are sub-networks of a larger network generated via Global Natural Products 771 Social Molecular Networking (GNPS). Nodes (small circles with $\mathrm{m} / \mathrm{z}$ values) represent unique 772 tandem mass spectrometry (MS/MS) consensus spectra and edges (lines) drawn between the 773 nodes correspond to similarity (cosine score) between MS/MS fragmentation. Annotation is 774 performed by MS/MS spectral library matching in GNPS platform. Pie charts within the 775 individual nodes qualitatively represent specific ion presence across groups: non-obese and non776 depressive, obese, depressive, and both obese and depressive symptom groups, as well as blank 777 samples. Molecular clusters 2, 3, 4, 5, 9, 17, 19, 30 and 34 represent structural diversity of 778 dipeptides. Molecular clusters 2, 14 and 26 represent aromatic amino acids tryptophan, tyrosine 779 and phenylalanine. 


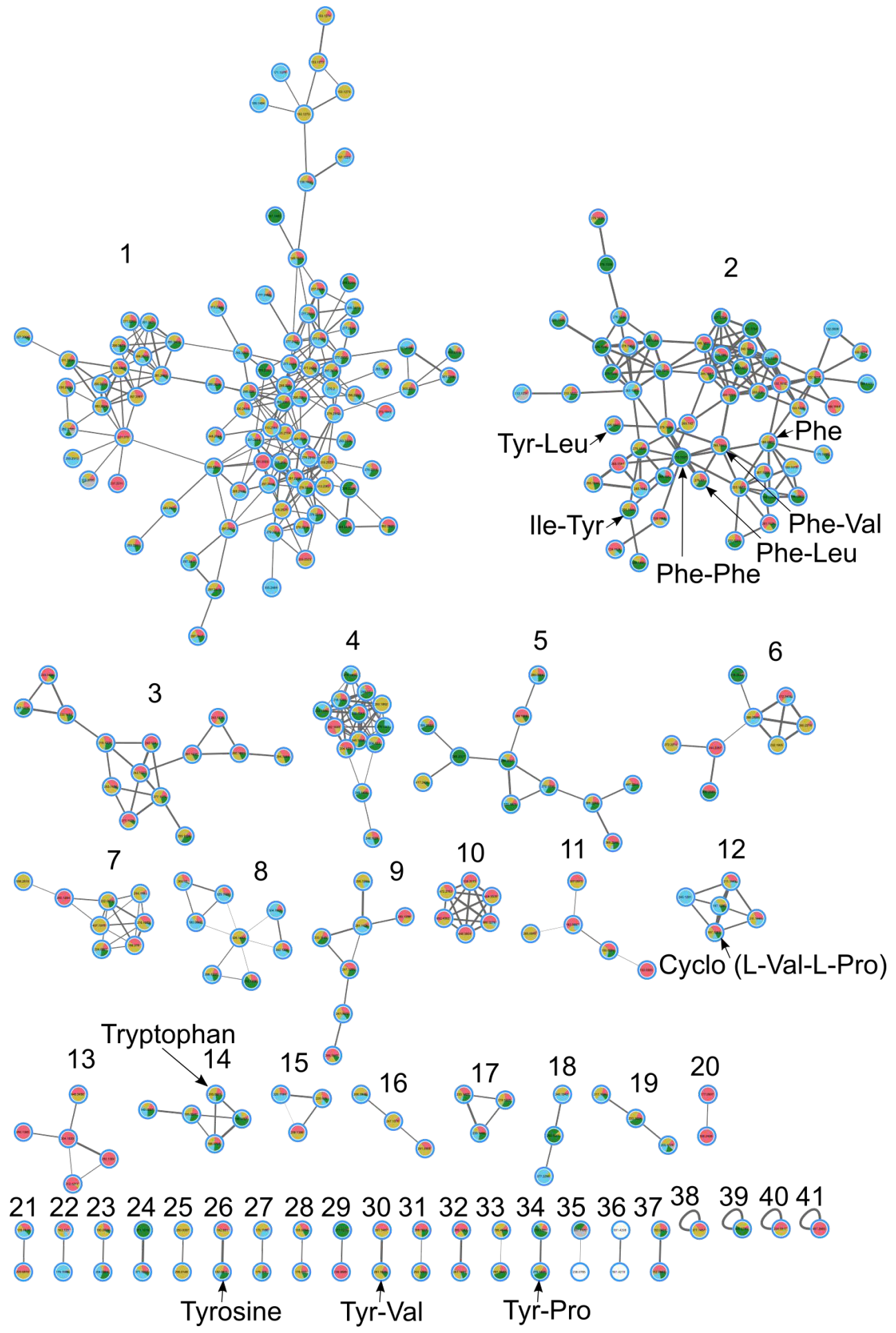

Pie chart colors

- Non-obese low-depressive Obese high-depressive

Non-obese high-depressive Obese low-depressive

Blank 
780 Figure 4. Differentially abundant molecular clusters and microbe-metabolite co-occurrences in 781 obesity-inflammation-depressive and inflammation status. (A) Sample plot showing log-ratio of 782 differential molecular features relative to cluster 1 (see left panel). The corresponding right 783 panels represent a scatterplot of samples showing log-ratio of differential features versus 784 inflammation status. Individual samples are colored by health status. Statistical significance of 785 the log-ratios was evaluated by pairwise comparisons using Wilcoxon rank sum test. A linear 786 regression model was employed for regressing log-ratios against BARIC values. (B) 787 Visualization of microbe-metabolite co-occurrences. Arrows represent microbes and dots 788 represent metabolites. The $x$ and $y$ axes represent principal components of the microbe789 metabolite conditional probabilities as determined by the neural network. Distances between 790 arrow tips quantify co-occurrence strengths between microbes, while directionality of the arrows 791 indicates which microbes and metabolites have a high probability of co-occurring. Only known 792 microbiota-derived molecules are labeled. Microbial abundances are estimated using differential 793 abundance analysis via multinomial regression. 
A

-Non-obsese low-depressive

- Non-obese high-depressive
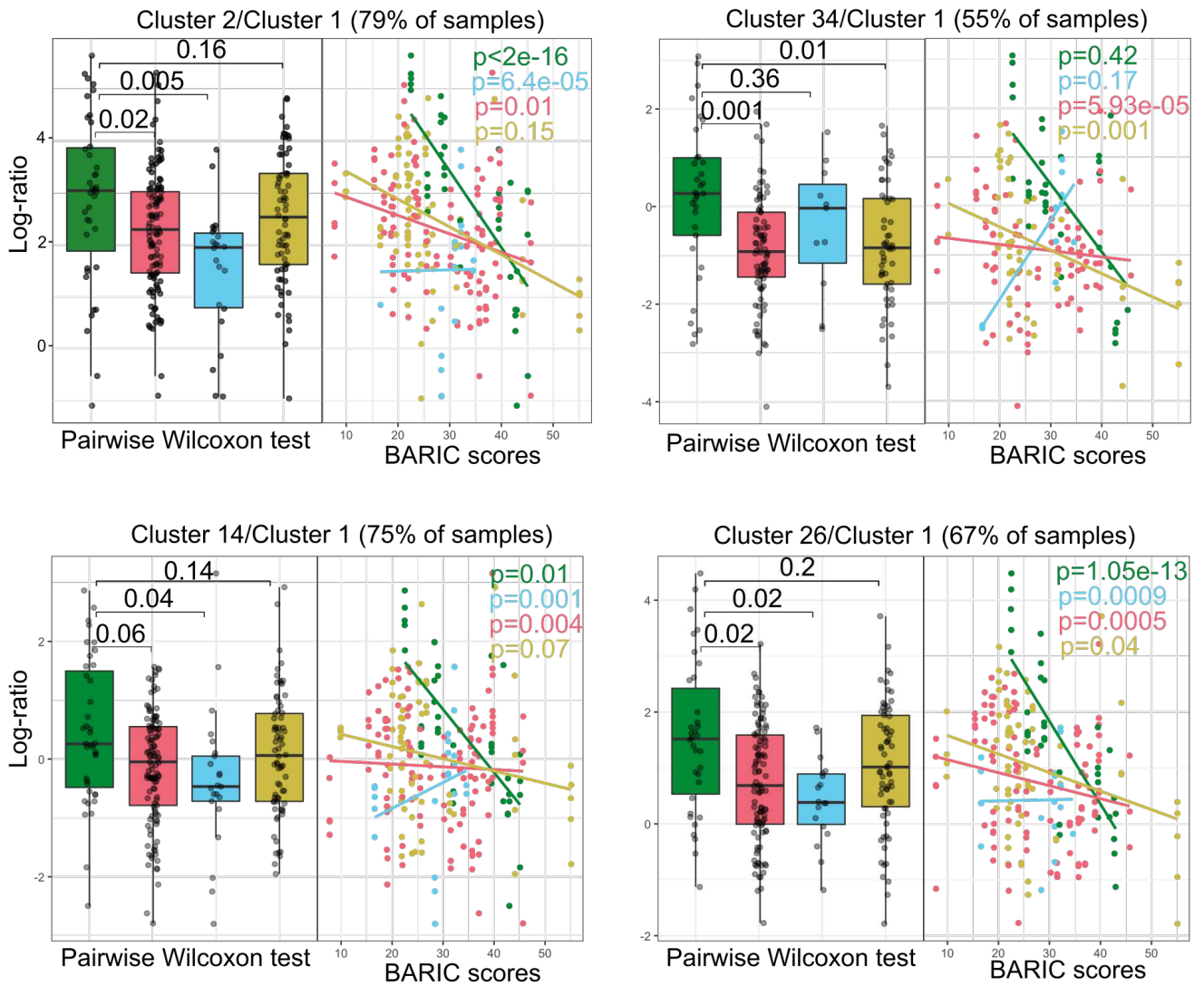

B

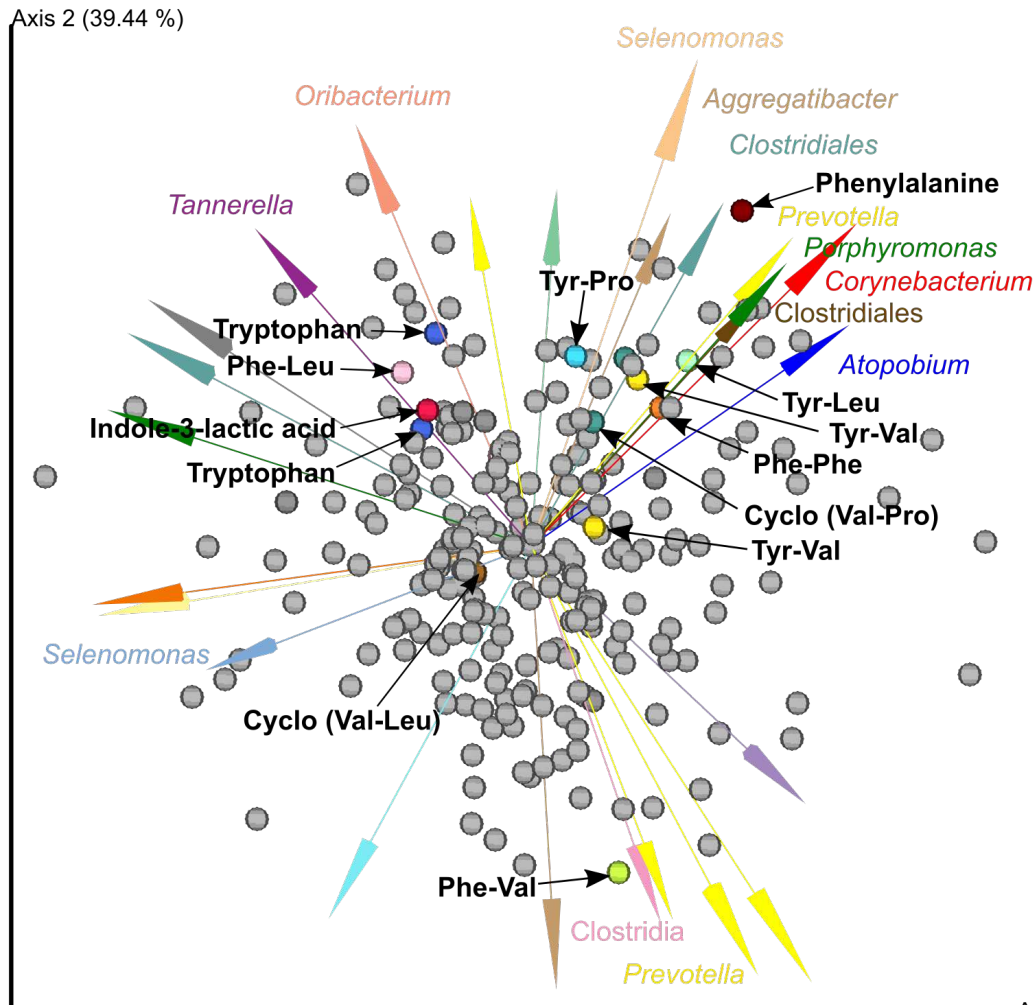


795 Table 1. Demographic and clinical characteristics of participants.

\begin{tabular}{lllll}
\hline Variable & $\begin{array}{l}\text { Non-obese low } \\
\text { depressive }^{\mathrm{a}}\end{array}$ & $\begin{array}{l}\text { Obese low } \\
\text { depressive }^{\mathrm{b}}\end{array}$ & $\begin{array}{l}\text { Non-obese high } \\
\text { depressive }^{\mathrm{c}}\end{array}$ & $\begin{array}{l}\text { Obese high } \\
\text { depressive }^{\mathrm{d}}\end{array}$ \\
\hline Age & $39 \pm 12.2$ & $38.9 \pm 17.2$ & $42.7 \pm 10.5$ & $43.5 \pm 10.9$ \\
Sex (\%female) & 44 & 50 & 61.1 & 73.3 \\
Race(\%C/AA/Asn/NS) & $72 / 16 / 12 / 0$ & $37.5 / 37.5 / 12.5 / 12.5$ & $55.6 / 16.7 / 27.8 / 0$ & $46.7 / 40 / 13.3 / 0$ \\
BARIC & $32.1 \pm 10.2^{\text {d }}$ & $21.9 \pm 6.2^{\text {c }}$ & $31.8 \pm 9^{\text {cd }}$ & $25.3 \pm 7.5^{\text {ac }}$ \\
BMI (kg/m2) & $25.1 \pm 2.9^{\text {bd }}$ & $35.5 \pm 4.7^{\text {ac }}$ & $26.6 \pm 2.9^{\text {bd }}$ & $36 \pm 4.7^{\text {ac }}$ \\
BDI-T & $0.5 \pm 0.8^{\text {cd }}$ & $0.6 \pm 0.7^{\text {cd }}$ & $7.9 \pm 5.4^{\text {ab }}$ & $7.9 \pm 5^{\text {ab }}$ \\
\hline
\end{tabular}

796 Values presented as mean \pm SD. Significant differences between groups were evaluated by

797 Mann-Whitney test and presented as superscripts. Abbreviations: $\mathrm{C}=$ Caucasian; AA = African-

798 American; Asn = Asian; NS = Mixed or not specified; BARIC $=$ monocyte beta-adrenergic

799 receptor-mediated inflammation control; BMI = body mass index; BDI-T $=$ Beck Depression

800 Inventory (BDI-Ia) total score.

Table 2. Beta-diversity analysis of $16 \mathrm{~S}$ derived ASVs across groups.

Unweighted-UniFrac Unweighted-UniFrac

\begin{tabular}{lll} 
Variable & $\mathrm{R}^{2}$ & $\mathrm{~F}$ \\
\hline Age & 0.01 & $3.88 * * *$ \\
Sex & 0.01 & $2.54 * * *$ \\
Race & 0.03 & $2.92 * * *$ \\
Time of day & 0.01 & 0.98 \\
BARIC & 0.02 & $4.71 * * *$ \\
Obesity & 0.008 & $0.004 * *$ \\
Depressive symptomatology & 0.01 & $0.001 * * *$ \\
Obesity-depressive symptomatology co-occurrences & 0.03 & $2.48 * * *$
\end{tabular}

Asteriks indicate statistical significance of PERMANOVA test, $\mathrm{p}<0.05$. 
801 Table 3. Post-hoc pairwise comparisons of beta-diversity between groups.

\begin{tabular}{lll} 
& $\begin{array}{l}\text { Unweighted- } \\
\text { UniFrac }\end{array}$ & $\begin{array}{l}\text { Unweighted- } \\
\text { UniFrac }\end{array}$ \\
Pairwise contrasts & $\mathrm{R}^{2}$ & $\mathrm{~F}$ \\
\hline Obese high-depressive x Non-obese low-depressive & 0.02 & $2.57 * * *$ \\
Obese low-depressive x Non-obese low-depressive & 0.02 & $1.91^{* *}$ \\
Non-obese high-depressive x Non-obese low-depressive & 0.04 & $2.4^{* *}$ \\
Obese low-depressive x Non-obese high-depressive & 0.02 & $2.05^{* *}$ \\
Obese low-depressive x Obese high-depressive & 0.01 & $2.19^{* * *}$ \\
Obese high-depressive x Non-obese high-depressive & 0.02 & $2.2^{* * *}$ \\
\hline
\end{tabular}




\section{Blood collection and cellular inflammation assay}

3 Blood samples were obtained for all participants after $12 \mathrm{~h}$ of fasting except for plain water and

4 collected in heparin anti-coagulant vacutainers (BD, Franklin Lakes, NJ). Cellular inflammation

5 regulation assays were performed on heparinized whole blood within $1 \mathrm{~h}$ of collection. Briefly,

$6200 \mathrm{pg} / \mathrm{mL}$ of lipopolysaccharide (LPS) (E.coli 0111:B4, catalog \#L4391, Sigma-Aldrich, St.

7 Louis, MO) was added to $300 \mu \mathrm{L}$ of blood in sterile 96-well polypropylene cell culture plates and

8 incubated for $30 \mathrm{~min}$ at $37^{\circ} \mathrm{C}$ with $5 \% \mathrm{CO}_{2}$. Media-treated samples served as controls. This

9 exogenous LPS dose was previously determined to elicit significant activation of monocytes,

10 with $30-90 \%$ producing TNF- $\alpha$ [1]. Monocyte beta-adrenergic receptor-mediated inflammation

11 control (i.e., "BARIC") was determined based on the inhibitory effect of isoproterenol (Iso), a

12 non-specific $\beta 1 / 2 \mathrm{AR}$ agonist, on monocytic intracellular TNF- $\alpha$ production in LPS-stimulated

13 blood as aforementioned. Briefly, LPS-stimulated blood was incubated with isoproterenol in $10^{-8}$

$14 \mathrm{M}$ final concentration and evaluated for intracellular monocyte TNF- $\alpha$ production using flow

15 cytometry, as previously described [1]. The proportion of CD14 ${ }^{+/ \operatorname{dim} H L A-D R^{+}}$(CD14: cat.

16 \#301808; HLA-DR: cat. \#307606, BioLegend, San Diego, CA) cells that were TNF- $\alpha^{+}$was

17 determined using FlowJo software (v10, TreeStar, Ashland, OR), and gates adjusted for TNF- $\alpha$ -

18 stained sample via fluorescence-minus-one controls [2,3]. Ultimately, BARIC was calculated as

19 the arithmetic difference in \%TNF- $\alpha+$ monocytes between LPS-treated and LPS + isoproterenol-

20 treated samples. Greater BARIC values indicate greater $\beta$-AR responsivity, and thus, better

21 Iso// - AR-mediated inflammation regulation. Smaller BARIC values may indicate impairment in 
23 cellular pathways that regulate inflammatory responses mediated by $\beta$-ARs (e.g., diminished

24 receptor sensitivity to agonists). BARIC measures monocytes responsivity to a $\beta$-AR agonist

25 during an inflammatory response to LPS. Reduced BARIC has been associated with

26 hypertension, cardiovascular disease risk factors, obesity, and higher serum cytokine levels [2,3].

\section{Saliva collection, DNA extraction and $16 \mathrm{~S}$ sequencing}

28 Saliva collection procedure and $16 \mathrm{~S}$ sequencing data was published previously [2]. However,

29 obesity-depressive symptom relationships were not previously investigated, and instead had

30 focused on temporal variation of the oral microbiota. Briefly, participants were provided with

31 Salivette (Sarstedt, \#51.1534, Nümbrecht, Germany) to roll the cotton Salivette inside the mouth

32 to stimulate salivation without chewing. Saturated Salivette was placed back into the tube by

33 mouth. Salivettes from each participant were collected at five time points across a single day:

34 waking, mid-morning (10:00 hrs), midday (12:00 hrs), afternoon (14:00 hrs), and evening (17:00

$35 \mathrm{hr}$ ). All waking samples were collected prior to oral hygiene activity, and ingestion of food or

36 drink. In addition, participants were instructed to abstain from consuming food or drinks other

37 than plain water for $30 \mathrm{~min}$ and to rinse their mouth with water prior to collection at all other

38 time points. Next, saliva was recovered from Salivette tubes by centrifuging at $1,000 \mathrm{x} \mathrm{g}$ for 2

39 minutes at $4^{\circ} \mathrm{C}$ and stored at $-80^{\circ} \mathrm{C}$. DNA from saliva samples was extracted by employing

40 Qiagen PowerSoil DNA kit as previously described [4]. V4 region of the 16S gene was amplified

41 according to the Earth Microbiome Project protocol $[5,6]$ and sequenced on the Illumina MiSeq

42 sequencing platform with a MiSeq Reagent Kit v2 and paired-end 150 bp cycles. 


\section{$43 \quad 16 \mathrm{~S}$ sequencing data processing}

44 Sequences were demultiplexed based on the barcode associated with each sample and sequence 45 quality control and ASV (Amplicon Sequence Variants) feature table construction was 46 conducted using the Deblur algorithm in QIIME2 (v.2018.4) [7]. Next, 223 potential sequencing

47 contaminants that appeared in both true and blank samples were removed from the ASV table

48 using decontam in $\mathrm{R}$ [8]. Low abundance features with fewer than 10 reads across samples and 49 singleton features present only in one sample were excluded. Taxonomy assignment was 50 performed by employing QIIME2 feature-classifier plugin with a pre-fit classifier [9] for the $5199 \%$ reference tree of Greengenes $13 \_8$ database. The output feature table contained an average 52 of 19,412 $\pm 9,187$ sequences per sample after removal of mitochondrial and chloroplast-derived 53 sequences. Multiple rarefactions were computed to a minimum depth of 1,122 reads to mitigate 54 uneven sequencing depth across samples. This resulted in 257 samples with 1,516 unique

55 features/ASVs and 455 unique taxa. Next, alpha-diversity indices Shannon diversity index and 56 Faith's Phylogenetic Diversity were calculated. Beta-diversity, was calculated using unweighted

57 UniFrac distance, which reflects presence-absence of taxa. We performed ordination on output 58 distance matrices using principal coordinates analysis ( $\mathrm{PCoA})$ and following visualization using 59 EMPeror plugin in QIIME2 [10].

\section{Small molecule metabolites detection through mass spectrometry}

61 Saliva was dried and resuspended in $80 \% \mathrm{MeOH}-20 \%$ water (Optima LC-MS grade; Fisher

62 Scientific, Fair Lawn, NJ, USA). Untargeted metabolomics was conducted with an ultrahigh-

63 performance liquid chromatography (Vanquish; Thermo Fisher Scientific, Waltham, MA, USA) 
64 system coupled to an orbitrap mass spectrometer (QExactive, Thermo Fisher Scientific). A C18

65 reversed-phase UHPLC column (Kinetex, 1.7- $\mu \mathrm{m}$ particles size, 50 x $2.1 \mathrm{~mm}$ ) (Phenomenex,

66 Torrance, CA, USA) was used for chromatographic separation. A linear gradient was applied as

67 follows: 0 to $0.5 \mathrm{~min}$, isocratic at $5 \%$ mobile phase (MP) B; 0.5 to $8.5 \mathrm{~min}, 100 \% \mathrm{MP} \mathrm{B} ; 8.5$ to

$6811 \mathrm{~min}$, isocratic at $100 \% \mathrm{MP} \mathrm{B} ; 11$ to $11.5 \mathrm{~min}, 5 \% \mathrm{MP} \mathrm{B} ; 11.5$ to $12 \mathrm{~min}, 5 \% \mathrm{MP}$ B, where

69 mobile phase $\mathrm{A}$ is water with $0.1 \%$ formic acid (vol/vol) and mobile phase $\mathrm{B}$ is

70 acetonitrile $-0.1 \%$ formic acid (vol/vol) (LC-MS grade solvents; Fisher Chemical). Electrospray

71 ionization in the positive mode was used. MS spectra were acquired in the mass range of $\mathrm{m} / \mathrm{z} 100$ 72 to 2,000 .

\section{MS1 feature finding and data processing}

74 Raw QExactive files were converted to .mzXML format using ProteoWizard tool MSConvert

75 [11] software. Data quality was assessed by evaluating the $\mathrm{m} / \mathrm{z}$ error and retention time of the 76 LC-MS standard solution (i.e., mixture of six compounds). MS1 feature finding was performed 77 in MZmine2 preprocessing workflow (MZmine-2.37.corr17.7_kai_merge2 version) available at 78 (https://github.com/robinschmid/mzmine2/releases) [12]. The mzMINE parameters used for 79 feature finding are as follows: mass detection (centroid; MS1, 1.5E3; MS2, 90); ADAP 80 Chromatogram builder (minimum group size in number of scans, 4; group intensity threshold, $815 \mathrm{E} 3$; minimum highest intensity, $2 \mathrm{E} 3 ; \mathrm{m} / \mathrm{z}$ tolerance, $0.001 \mathrm{~m} / \mathrm{z}$ to $20 \mathrm{ppm}$ ); chromatogram 82 deconvolution (local minimum search, chromatographic threshold of 96\%, search minimum in 83 retention time [RT] range [minutes] of 0.03 , minimum relative height of $5 \%$, minimum absolute 84 height of 2E3, minimum ratio of peak top/edge of 1 and peak duration range [minutes] of 0 to 
$852 ; \mathrm{m} / \mathrm{z}$ center calculation set to auto; $\mathrm{m} / \mathrm{z}$ range for MS2 scan pairing (daltons) of 0.02 and RT

86 range for MS2 scan pairing (minutes) of 0.15$)$; isotope peaks grouper $(\mathrm{m} / \mathrm{z}$ tolerance set to

$870.0015 \mathrm{~m} / \mathrm{z}$ or $10 \mathrm{ppm}$; retention time tolerance of 0.05 , maximum charge of 3 ; and

88 representative isotope set to most intense); order peak lists; join aligner ( $\mathrm{m} / \mathrm{z}$ tolerance set at

$890.0015 \mathrm{~m} / \mathrm{z}$ or $15 \mathrm{ppm}$; weight for $\mathrm{m} / \mathrm{z}$ of 2 ; retention time tolerance of $0.2 \mathrm{~min}$; weight for RT of

90 1. A filter was used such that only features present in at least two samples were included.

\section{Feature based mass spectral molecular networking (FBMN)}

92 The output of aforementioned workflow, a data matrix of MS1 features that triggered MS2 scans

93 by sample (.mgf and .csv quant table), were uploaded along with the metadata file to Global

94 Natural Product Social Molecular Networking (GNPS) (https://gnps.ucsd.edu) [13,14]. Feature-

95 based molecular networking (version release_20) [15] was performed, and library IDs were

96 generated. Molecular networking parameters were set as follows: precursor ion mass tolerance

97 and fragment ion tolerance of $0.02 \mathrm{Da}$ to cluster consensus spectra; the minimum score between

98 a pair of MS2 consensus spectra was set at 0.7 and 6 as the minimum number of ions matched as

99 described at https:/gnps.ucsd.edu/ProteoSAFe/status.jsp?

100 task=f192a0030f694224a0ba8f08223a1323. The molecular network output from GNPS was then

101 uploaded to Cytoscape (version 3.5.1 http://www.cytoscape.org/) [16], for advanced

102 visualization. Nodes were labelled with spectral matches to GNPS with $\mathrm{m} / \mathrm{z}$ values, and edge

103 thickness is proportional to the cosine score. 


\section{Supplementary figures}

105 Figure S1. Matrix of plots illustrating Pearson correlations among obesity, depressive

106 symptoms, inflammation and sex, across participants. Histograms of the variables displayed

107 along the matrix diagonal represent distribution of samples and scatter plots of variable pairs are

108 displayed in the off diagonal. Correlation coefficients displayed represent the slopes of the least-

109 squares reference lines in the scatter plots.

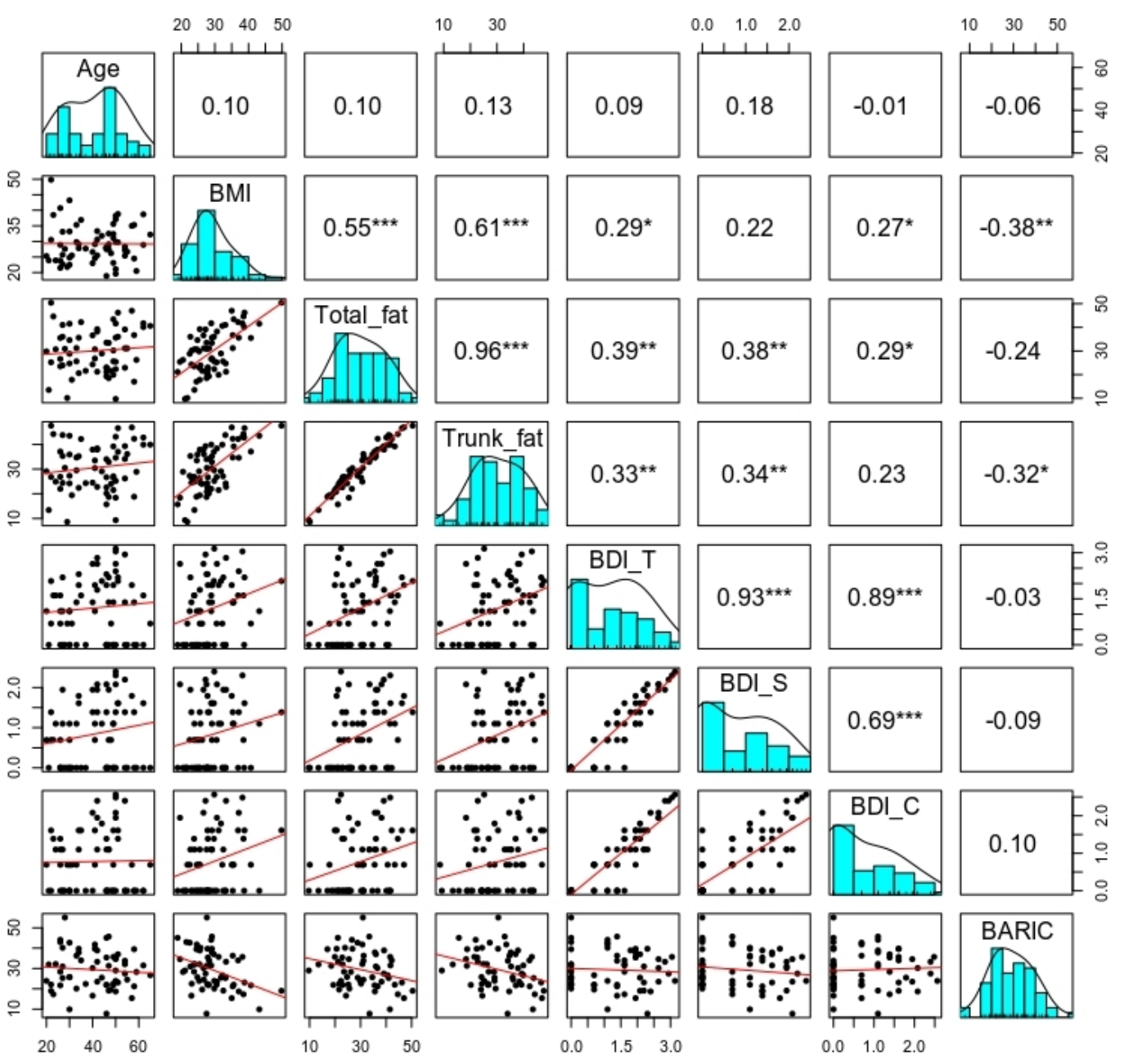


110 Figure S2. Per sample based RF analysis. (A), Receiver operating characteristic curves

111 (AUROC) illustrating classification accuracy of the random forest model across all groups (i.e.

112 controls, Ob/lower Dep, Non-ob/higher-Dep, Ob/higher-Dep) and (B), Area under precision

113 recall curves (AUPRC) illustrating performance of the random forest model across all groups.

A

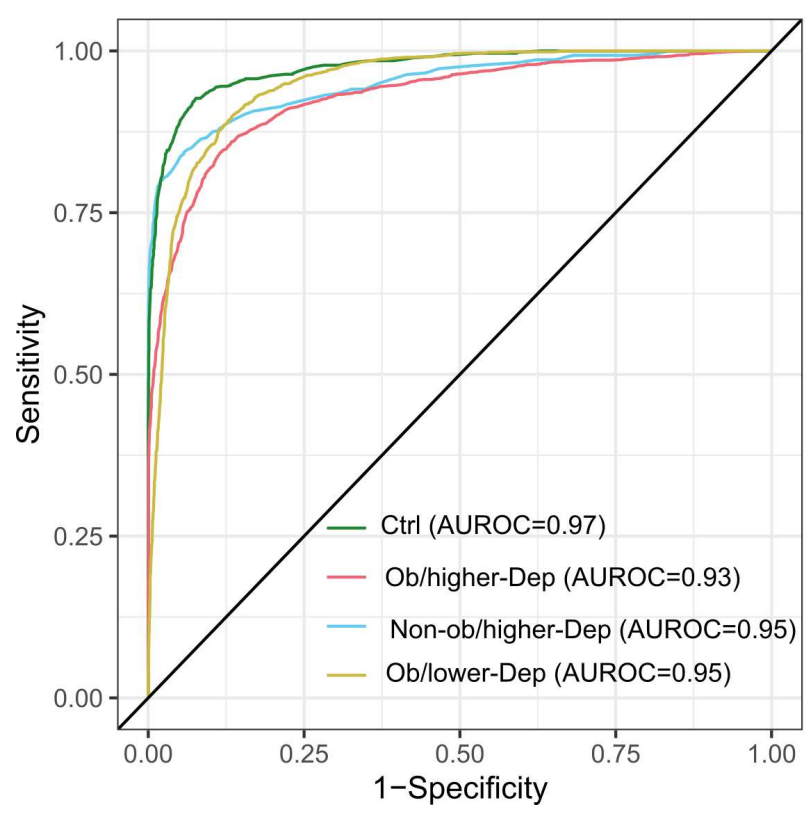

B

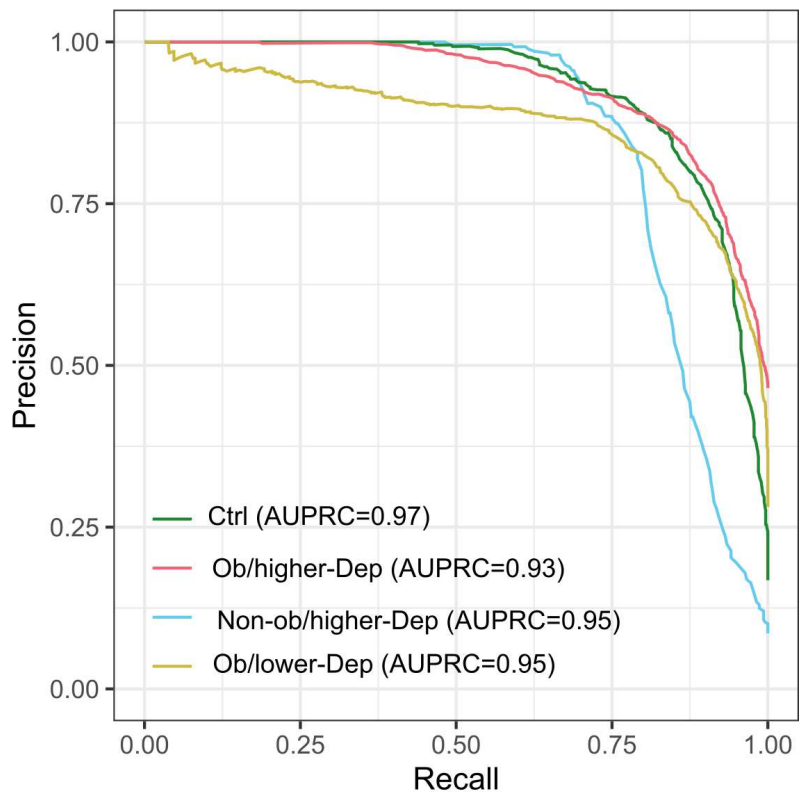


114 Figure S3. Chemical diversity captured in salivary metabolomes. Branches in the circular

115 chemical tree are colored according to the class type and branch labels represent putatively

116 annotated chemical features at subclass level based on chemical taxonomy. Bar graphs at the leaf

117 tips illustrate relative abundance of molecules across groups.

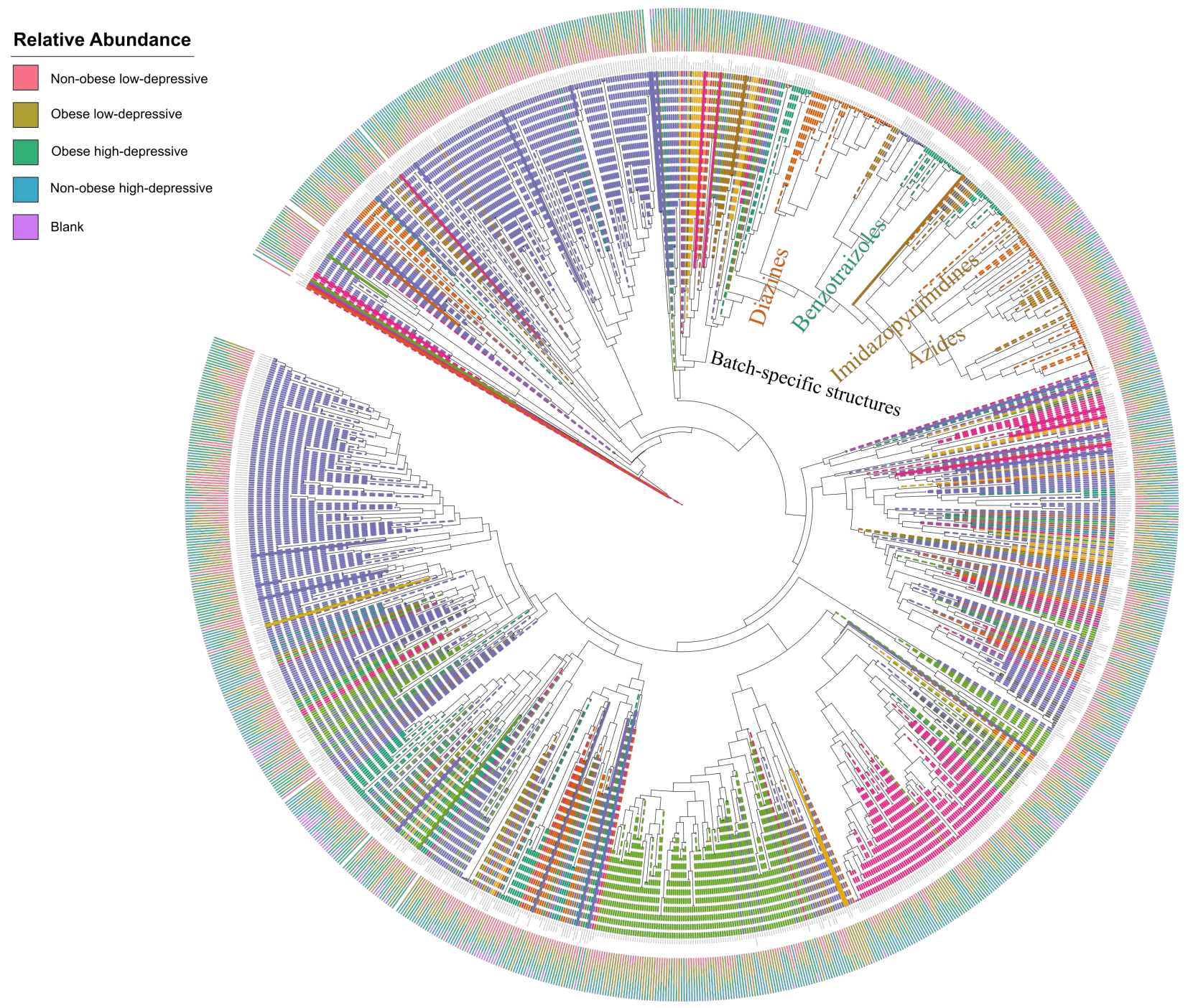




\section{References}

119

120 1. Hong S, Dimitrov S, Cheng T, Redwine L, Pruitt C, Mills PJ, et al. Beta-adrenergic receptor

121 mediated inflammation control by monocytes is associated with blood pressure and risk factors

122 for cardiovascular disease. Brain Behav Immun. Academic Press Inc.; 2015;50:31-8.

123 2. Kohn JN, Cabrera Y, Dimitrov S, Guay-Ross N, Pruitt C, Shaikh FD, et al. Sex-specific roles 124 of cellular inflammation and cardiometabolism in obesity-associated depressive 125 symptomatology. Int J Obes. Nature Publishing Group; 2019;43:2045-56.

126 3. Dimitrov S, Hulteng E, Hong S. Inflammation and exercise: Inhibition of monocytic 127 intracellular TNF production by acute exercise via $\beta 2$-adrenergic activation. Brain Behav 128 Immun. Academic Press Inc.; 2017;61:60-8.

129 4. Marotz C, Amir A, Humphrey G, Gaffney J, Gogul G, Knight R. DNA extraction for 130 streamlined metagenomics of diverse environmental samples. Biotechniques. Eaton Publishing 131 Company; 2017;62:290-3.

132 5. Thompson LR, Sanders JG, McDonald D, Amir A, Ladau J, Locey KJ, et al. A communal 133 catalogue reveals Earth's multiscale microbial diversity. Nature. Nature Publishing Group; 134 2017;551:457-63.

135 6. Walters W, Hyde ER, Berg-Lyons D, Ackermann G, Humphrey G, Parada A, et al. Improved 136 Bacterial 16S rRNA Gene (V4 and V4-5) and Fungal Internal Transcribed Spacer Marker Gene 137 Primers for Microbial Community Surveys. mSystems. American Society for Microbiology; $138 \quad 2016 ; 1: 9-15$.

139 7. Bolyen E, Rideout JR, Dillon MR, Bokulich NA, Abnet CC, Al-Ghalith GA, et al. 140 Reproducible, interactive, scalable and extensible microbiome data science using QIIME 2. Nat. 141 Biotechnol. Nature Publishing Group; 2019. p. 852-7.

142 8. Davis NM, Proctor DiM, Holmes SP, Relman DA, Callahan BJ. Simple statistical 143 identification and removal of contaminant sequences in marker-gene and metagenomics data. 144 Microbiome. BioMed Central Ltd.; 2018;6.

145 9. Bokulich NA, Kaehler BD, Rideout JR, Dillon M, Bolyen E, Knight R, et al. Optimizing 146 taxonomic classification of marker-gene amplicon sequences with QIIME 2's q2-feature147 classifier plugin. Microbiome. BioMed Central Ltd.; 2018;6.

148 10. Vázquez-Baeza Y, Pirrung M, Gonzalez A, Knight R. EMPeror: A tool for visualizing high149 throughput microbial community data. Gigascience. BioMed Central Ltd.; 2013;2.

150 11. Chambers MC, MacLean B, Burke R, Amodei D, Ruderman DL, Neumann S, et al. A cross- 
151 platform toolkit for mass spectrometry and proteomics. Nat. Biotechnol. Nat Biotechnol; 2012. 152 p. 918-20.

153 12. Pluskal T, Castillo S, Villar-Briones A, Orešič M. MZmine 2: Modular framework for 154 processing, visualizing, and analyzing mass spectrometry-based molecular profile data. BMC 155 Bioinformatics. BMC Bioinformatics; 2010;11.

156 13. Aron AT, Gentry EC, McPhail KL, Nothias LF, Nothias-Esposito M, Bouslimani A, et al. 157 Reproducible molecular networking of untargeted mass spectrometry data using GNPS. Nat 158 Protoc. Nature Research; 2020;15:1954-91.

159 14. Wang M, Carver JJ, Phelan V V., Sanchez LM, Garg N, Peng Y, et al. Sharing and 160 community curation of mass spectrometry data with Global Natural Products Social Molecular 161 Networking. Nat. Biotechnol. Nature Publishing Group; 2016. p. 828-37.

162 15. Nothias LF, Petras D, Schmid R, Dührkop K, Rainer J, Sarvepalli A, et al. Feature-based 163 molecular networking in the GNPS analysis environment. Nat Methods. Nature Research; $1642020 ; 17: 905-8$.

165 16. Shannon P, Markiel A, Ozier O, Baliga NS, Wang JT, Ramage D, et al. Cytoscape: A 166 software Environment for integrated models of biomolecular interaction networks. Genome Res. 167 Genome Res; 2003;13:2498-504.

168 


\section{Supplementary Files}

This is a list of supplementary files associated with this preprint. Click to download.

- Supplementarytables.xlsx 